\title{
Mechanical characterization and constitutive parameter identification of anisotropic tubular materials for hydroforming applications
}

\author{
Ali Khalfallah a,b,*, Marta Cristina Oliveira a , José Luís Alves ${ }^{\mathrm{c}}$, Temim Zribi ${ }^{\mathrm{b}}$, \\ Hédi Belhadjsalah ${ }^{\mathrm{b}}$, Luís Filipe Menezes ${ }^{\mathrm{a}}$ \\ ${ }^{a}$ CEMUC, Department of Mechanical Engineering, University of Coimbra, Polo II, Rua Luís Reis Santos, Pinhal de Marrocos, 3030-788 Coimbra, Portugal \\ ${ }^{\mathrm{b}}$ LGM, Department of Mechanical Engineering, Ecole Nationale d'Ingénieurs de Monastir, Av Ibn ElJazzar, 5019 Monastir, Tunisia

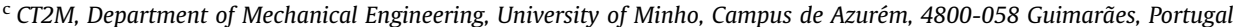

\section{A R T I C L E I N F O}

Article history:

Received 24 April 2015

Received in revised form

15 September 2015

Accepted 29 September 2015

Available online 8 October 2015

\section{Keywords:}

Tube hydroforming

Material characterization

Parameter identification

Anisotropy

FEA

\begin{abstract}
A B S T R A C T
This paper aims to identify the constitutive parameters of anisotropic tubular materials and to verify the accuracy of models' prediction. The identification of the constitutive parameters is based on information obtained from tensile tests, performed on samples cut from the tubes, and from the free tubular bulge test, using a home-developed bulge forming machine. Two tubular materials exhibiting different anisotropic behaviour and work hardening characteristics are investigated: a mild steel S235 seamed tube and an aluminium alloy AA6063 extruded tube. It is shown that advanced phenomenological yield functions, including a large number of anisotropy parameters, can accurately describe the plastic flow of highly anisotropic tubular materials during the tube hydroforming process. However, parameter identification procedure of advanced yield criteria requires a high number of experimental tests. Thus, in order to enable the parameter identification of these yield criteria when using a reduced set of experimental results, the present study develops a method that combines tensile tests with (i) a free bulge test, which is used to characterize the biaxial stress state experienced by the tube during the bulge testing, and (ii) some generated artificial input data. Finally, the proposed method shows an excellent agreement between numerical predictions and experimental results.
\end{abstract}

(c) 2015 Elsevier Ltd. All rights reserved.

\section{Introduction}

Tube hydroforming is one of the modern metal forming processes which is becoming a widespread manufacturing process in automotive, aircraft and aerospace industries, to produce lightweight parts with complex shapes [1-3], aiming at reducing energy consumption and decreasing gas emissions in the field of transports. Compared to other conventional manufacturing processes, such as stamping combined with welding technologies, tube hydroforming provides complex and non-uniform cross section parts having better consolidation, tighter tolerances, improved structural strength and stiffness and lower manufacturing costs, resulting from reduced number of auxiliary forming and assembly operations $[1,4]$.

However, tube hydroforming is technologically a very complex process, mainly due to the strongly anisotropic with limited formability behaviour of tubular materials, friction between workpiece and

\footnotetext{
* Corresponding author at: CEMUC, Department of Mechanical Engineering, University of Coimbra, Polo II, Rua Luís Reis Santos, Pinhal de Marrocos, 3030-788 Coimbra, Portugal. Tel.: +351 239 790700; fax: +351 239790701.

E-mail addresses: ali.khalfallah@dem.uc.pt, ali.khalfallah@gmail.com (A. Khalfallah).
}

die, process parameter control difficulties, and among others $[5,6]$. Moreover, the plastic behaviour is usually determined by simple analytical approaches which have led to limited understanding of the process $[7,8]$. Fortunately, finite element (FE) analysis has been more and more used to study and deepen our understanding about sheet forming processes in general, and the tube hydroforming process in particular. Several process parameters playing a paramount role on achieving successful tube hydroforming operations have been studied by FEA, amongst others the material properties and the constitutive parameters identified from experimental tests: unquestionably a wellcharacterized material model enhances the accuracy of numerical simulations of the tube hydroforming process [9].

In general, tubular material properties are identified from tensile tests carried out either on samples cut from raw sheet materials prior to roll forming and welding operations or on specimens cut from flat sheet obtained from roll-formed tube [10,11]. In fact, when tensile samples are cut off from a sheet metal obtained from flattened rollformed tube, formability and flow stress of the tubular blank are different from those extracted from initial rolled sheet metal, since it has been already pre-strained, firstly by the roll-forming and then by the flattening operation. On the other hand, tubular material properties can be extracted from tensile tests performed on samples cut off 
from the tube along axial direction [12]. Nevertheless, in such case, the material properties are restricted to the longitudinal direction. Although, the tensile test carried out in a single direction is generally insufficient for identifying the constitutive parameters of anisotropic tubular materials, it can be used to describe the work-hardening behaviour of the material up to the onset of tensile instability. However, plastic deformation mode in tensile testing is uniaxial, whereas in tube hydroforming process, the tube undergoes primarily biaxial stress loadings [10]. Accordingly, tensile tests are limited in the description of the plastic behaviour of tubular materials. Alternatively, the free-expansion test, usually called free bulge test, has begun to be commonly used for the mechanical characterization of tubular materials subjected to tube hydroforming process [13,14]. The free bulge test offers a testing method which imitates, as closely as possible, the loading conditions of real tube hydroforming operations. In order to identify the material properties from the free bulge test, the applied internal pressure plotted against the pole height curve is required; the free bulge testing is, thus, the preferable and recommended method for the mechanical characterization of tubes utilized in hydroforming process [9,15].

Several analytical models have been reported in the literature to determine the stress-strain relationship of tubular materials. The analytical approaches are based on the plastic membrane theory, the force equilibrium equations and the assumption of specific geometrical profiles (i.e. circular and elliptical profiles) encountered in the free bulge test. Fuchizawa proposed a method that uses the internal pressure and the bulge height at the pole of the free bulge zone, considered as a circular profile [9]. Sokolowsky et al. [16] developed a simple method, similar to the one proposed by Fuchizawa, based on the thin-walled theorem to evaluate the tubular material characteristics. Koç et al. [9] investigated a number of methods to record the parameters needed for the determination of the flow stress curves, measured either online or off-line. Hwang et al. [17] put forward a mathematical model to deduce the meridian radius of curvature and thickness distribution by considering the bulged zone in the centre of the dome as an elliptical profile. Bortot et al. [18] developed a method for the on-line measurement of geometrical features using coordinate measuring apparatus. Valesco et al. [19] proposed an analytical approach for tube bulging tests based on geometric observations, assuming a circular profile of the bulged zone and ignoring the fillet radius of the tube guiding dies. Lianfa et al. [20] Saboori et al. [8] and Liu et al. [21] have determined the flow stress curves for a diversity of tubular materials, using approaches based on the evaluation of the meridian radius of curvature by means of curve-fitting least squares methods. It is worth noting that the aforementioned methods assume material's isotropy, disregarding the studied tubular materials' anisotropy. Besides, there are other investigations proposing to take into account the tube's anisotropy. Among others, Fuchizawa et al. [22] investigated the influence of plastic anisotropy on the deformation of tubes in bulge forming, while Hwang has extended the isotropic calculation method proposed in [23], considering the anisotropy effects $[17,24]$. However, all these studies concerning the determination of flow stress curves of tubular materials consider the assumption of some hypothesis to simplify the analytical models. Thus, the main drawbacks of these simplified analytical models can be summarized as follows: (i) elastic deformation is ignored; (ii) despite the apparent strongly anisotropic behaviour of tubular materials, such as for extruded tubes, isotropy is commonly assumed for determining the stress-strain relationship; (iii) bending effects are neglected; (iv) simple geometric profiles of the bulged zone (i.e. circular or elliptical profiles) are supposed; (v) homogeneity assumption of stress and strain fields; and (vi) plane stress state is also considered (i.e., through-thickness normal stress is disregarded). Despite of this, Smith et al. [25] have demonstrated that the plane stress assumption may not be proper when very high pressure is applied.

An accurate identification of constitutive parameters is an utmost step for reliable FE simulation results. In addition, uncertainties related with experimental procedures (e.g. imprecision on initial thickness and radius of the tube, sensors positioning,...) must be well estimated and established [26]. In order to overcome the limitation of analytical models, inverse methods are considered to accomplish reliable identification of constitutive material parameters. Recently, Zribi et al. [27,28] have identified the flow stress and anisotropy parameters using a hybrid experimental-numerical method based upon both the free bulge test and uniaxial tensile tests. Strano et al. [29] have suggested an inverse energy approach to determine the flow stress of tubular materials, assuming isotropic behaviour. Xu et al. [30] proposed an adaptive inverse finite element method for tubular materials. Freshly, we have carried out a comparative study between two analytical models and an inverse method which are used for parameter identification of four tubular materials. We have shown the restricted ability of analytical models for the determination of accurate flow stress curves, compared to inverse method [7]. However, the calibrated constitutive equations using inverse method are limited to the von Mises or the Hill48 yield criteria, which are insufficient for modelling highly anisotropic plastic behaviour of arbitrary tubular materials, such as steels and extruded aluminium alloys [31].

The present study puts forward a method using a reduced set of uniaxial tensile tests and the free bulge test to calibrate advanced yield criteria, for the description of anisotropic plastic behaviour of low carbon tube steel S235 and extruded aluminium alloy AA6063. Three yield criteria are used to model the anisotropic plastic behaviour of tubes, namely, Hill48 [32], Yld91 [33] and CB2001 [34] yield criteria. In addition, von Mises isotropic yield function was as well used for comparison purposes. The identified parameters are used to simulate the free bulge test. The predicted bulge height $v$ s. internal pressure curve, thickness distribution and axial bulge profiles are compared to experimental results.

Finally, the present work aims at demonstrating that improved results can be obtained using advanced yield criterion, such as, CB2001 yield criterion, even if solely a reduced set of experimental data is available. Artificial data can be generated from less flexible, but robust yield functions and constitute a mean of compensating the missing experimental input data compulsory for successful identification of advanced yield criteria, in order to decrease/ minimize the cost of the experimental work associated with the parameter identification.

\section{Experiments and results}

\subsection{Materials}

Free bulge forming tests were performed on tubes made of low carbon steel S235 and aluminium alloy AA6063. The steel tube is a thin-walled seam welded tube by high frequency and the aluminium alloy tube is a circular extruded profile. The tube's external diameter is $50 \mathrm{~mm}$ with initial wall-thickness of $1.07 \mathrm{~mm}$ for the low carbon steel tube and $2.04 \mathrm{~mm}$ for the aluminium alloy.

\subsection{Uniaxial tensile tests}

The mechanical characterization of tubes by uniaxial tensile tests is achieved so as to measure the anisotropy Lankford coefficients ( $r$-values) and the flow stresses. By opening and flattening tubes, sheets of both materials are obtained and tensile specimens are then cut off in three directions, i.e. $0^{\circ}, 45^{\circ}$ and $90^{\circ}$ with respect 
to the referential (or axial) direction. Tensile specimens were machined from the flattened tubes with respect to ASTM: E8/E8M standards. Tensile tests were conducted at room temperature and tensile samples were extended to failure in a Shimadzu universal materials testing machine at a stroke rate of $5 \mathrm{~mm} / \mathrm{min}$ and the longitudinal and transversal displacements were measured in the gauge zone by longitudinal and transversal extensometers (LVDTs). For each specimen's orientation, at least three samples were cut off and tested to ensure accuracy and the test reproducibility. An average of the three tensile curves at each direction was calculated to represent the material properties in each direction where the specimen was cut off. The material anisotropic properties are obtained from Lankford coefficients measured from tensile tests. The Lankford anisotropy coefficient $r$-value is defined as the ratio of the strain in the width direction to that in the thickness direction. Thus, $r$-value represents the slope value of the best linear fitting of the thickness strain against the width strain. Using plasticity volume constancy, longitudinal and transversal displacements are used to calculate thickness and width strains.

In this work, the plastic flow (i.e. the isotropic hardening) is described considering only the stress-strain curve obtained from specimens cut off from tubes and oriented along the tubes' axial (or referential) direction. Besides, a set of experimental data which consists of $3 r$-values and 3 initial yield stresses, determined for the three directions with respect to the referential direction was acquired. However, it is well-known that initial yield stresses are affected by the flattening operation. Thus, in order to reduce inaccuracies on experimental initial yield stresses, as shown by Zribi et al. [28], the stresses along the three directions were measured at an equivalent plastic strain of $10 \%$. Even though, measured Lankford coefficients ( $r$-values) are assumed to insignificantly be affected by the tube flattening operation.

Tables 1 and 2 present the experimental $r$-values and flow stresses (at $\bar{\varepsilon}^{\mathrm{pl}}=10 \%$ ) for S235 and AA6063 tubular materials, respectively. By analysing these latter tables, it is noticed the differing anisotropic behaviour of the two tubes, as revealed by either the normal or the planar anisotropy coefficients. Additionally, it can also be seen the more isotropic plastic character of the flow stresses in case of the steel, compared to the extruded aluminium alloy. This remarkable anisotropy of the aluminium alloy behaviour, might probably associated to the extrusion process itself, in which the referential direction coincides with the extrusion direction and the $90^{\circ}$ with the circumferential one.

\subsection{Free tube hydroforming tests}

Self-designed and manufactured tube hydroforming machine is used to carry out tube hydroforming tests. Fig. 1(a) shows a photo of the apparatus for bulge forming operations [28]. This new equipment has the merits of strong flexibility in use and it is stand-alone tool. This avoids the use of a hydraulic press to close up the dies. The tube is placed between two die-halves and its ends are strapped up against the chamfers by pushing inward the actuators to lock the tube ends to avoid the hydraulic fluid leakage. There are holes through both actuators so that the fluid can be pumped by means of a hydraulic

Table 1

Experimental uniaxial tensile data for the low carbon steel S235.

\begin{tabular}{llllll}
\hline $\begin{array}{l}\text { Angle } \\
\text { from } \\
\text { RD } \\
(\mathrm{deg})\end{array}$ & $r$-Value (-) & $\begin{array}{l}\text { Flow stress at } \\
\bar{\varepsilon}^{p l}=10 \%(\mathrm{MPa})\end{array}$ & $\begin{array}{l}\text { Normalized } \\
\text { flow stress }\end{array}$ & $\begin{array}{l}\text { Normal } \\
\text { anisotropy } \\
\text { coefficient } \\
\bar{r}\end{array}$ & $\begin{array}{l}\text { Planar ani- } \\
\text { sotropy } \\
\text { coefficient } \\
\Delta r\end{array}$ \\
\hline 0 & $1.48 \pm 0.06$ & $442.9 \pm 5$ & 1.000 & 1.20 & 0.57 \\
45 & $0.92 \pm 0.05$ & $467.4 \pm 5$ & 1.055 & & \\
90 & $1.51 \pm 0.07$ & $438.2 \pm 5$ & 0.989 & & \\
\hline
\end{tabular}

Table 2

Experimental uniaxial tensile data for the AA6063 aluminium alloy.

\begin{tabular}{|c|c|c|c|c|c|}
\hline $\begin{array}{l}\text { Angle } \\
\text { from } \\
\text { RD } \\
\text { (deg) }\end{array}$ & $r$-Value $(-)$ & $\begin{array}{l}\text { Flow stress at } \\
\bar{\varepsilon}^{p l}=10 \%(\mathrm{MPa})\end{array}$ & $\begin{array}{l}\text { Normalized } \\
\text { flow stress }\end{array}$ & $\begin{array}{l}\text { Normal } \\
\text { anisotropy } \\
\text { coefficient } \\
\bar{r}\end{array}$ & $\begin{array}{l}\text { Planar ani- } \\
\text { sotropy } \\
\text { coefficient } \\
\Delta r\end{array}$ \\
\hline 0 & $0.59 \pm 0.04$ & $112.8 \pm 3$ & 1.000 & 0.76 & -0.14 \\
\hline 45 & $0.83 \pm 0.04$ & $77.4 \pm 3$ & 0.686 & & \\
\hline 90 & $0.79 \pm 0.06$ & $81.8 \pm 3$ & 0.725 & & \\
\hline
\end{tabular}

\section{a}

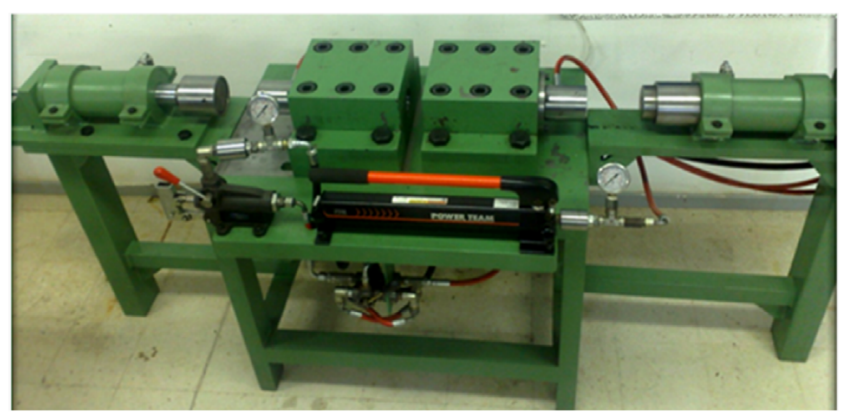

b

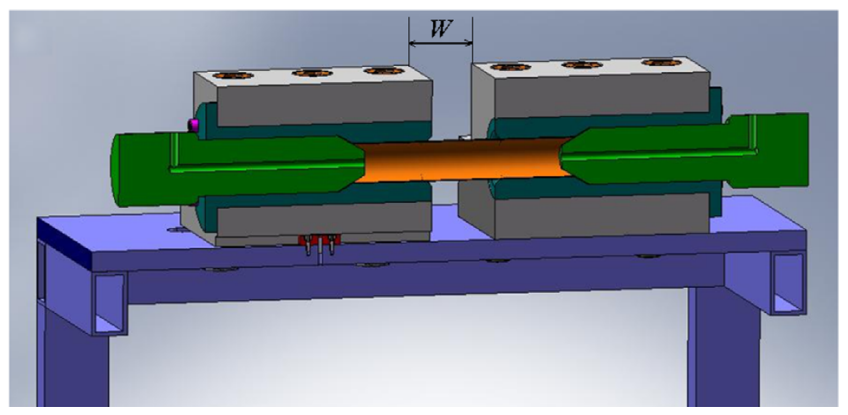

Fig. 1. Tube hydroforming equipment: (a) testing machine [28] and (b) CAD model.

pump to build up the pressure inside the tube. Fig. 1(b) shows the CAD model to more clarify this description. By translating the position of the movable die (i.e., die on the right), the length of the tube bulged region can be adjusted to achieve hydroformed parts with different lengths. Also it allows the use of different cross-section shaped dies [28]. Fig. 2 shows samples of hydroformed tubes with various width lengths and also displays the conical shapes at the ends of the tubes obtained at the first stage of the bulge test to guarantee an effective sealing and to tightly attach the tube extremities against the die chamfers. This tube hydroforming equipment was designed and manufactured to bulge several tubular materials, allowing an internal pressure up to approximately $100 \mathrm{MPa}$.

This tooling was used to carry out free bulge tests on tubes made of S235 and AA6063 materials. The width of the bulged area $W$ is set to the value of $60 \mathrm{~mm}$ to attain a maximum bulge height. The die entrance radius $R_{d}$ is set equal to $7.5 \mathrm{~mm}$ as shown in Fig. 3.

Two sets of experimental bulging tests were carried out. Firstly, the free bulge test is performed continuously by increasing the internal pressure until tube fracture. The forming pressure is recorded simultaneously along with the bulge height at the pole of the dome by using pressure transducer and inductive displacement transducer, plugged in an acquisition system which is connected to a PC. Secondly, stepwise free bulge tests were conducted on several tube samples. Each tube was bulged at a given pressure level. The experimental hoop strain and the wall thickness at the pole dome were measured for the corresponding pressure levels. 

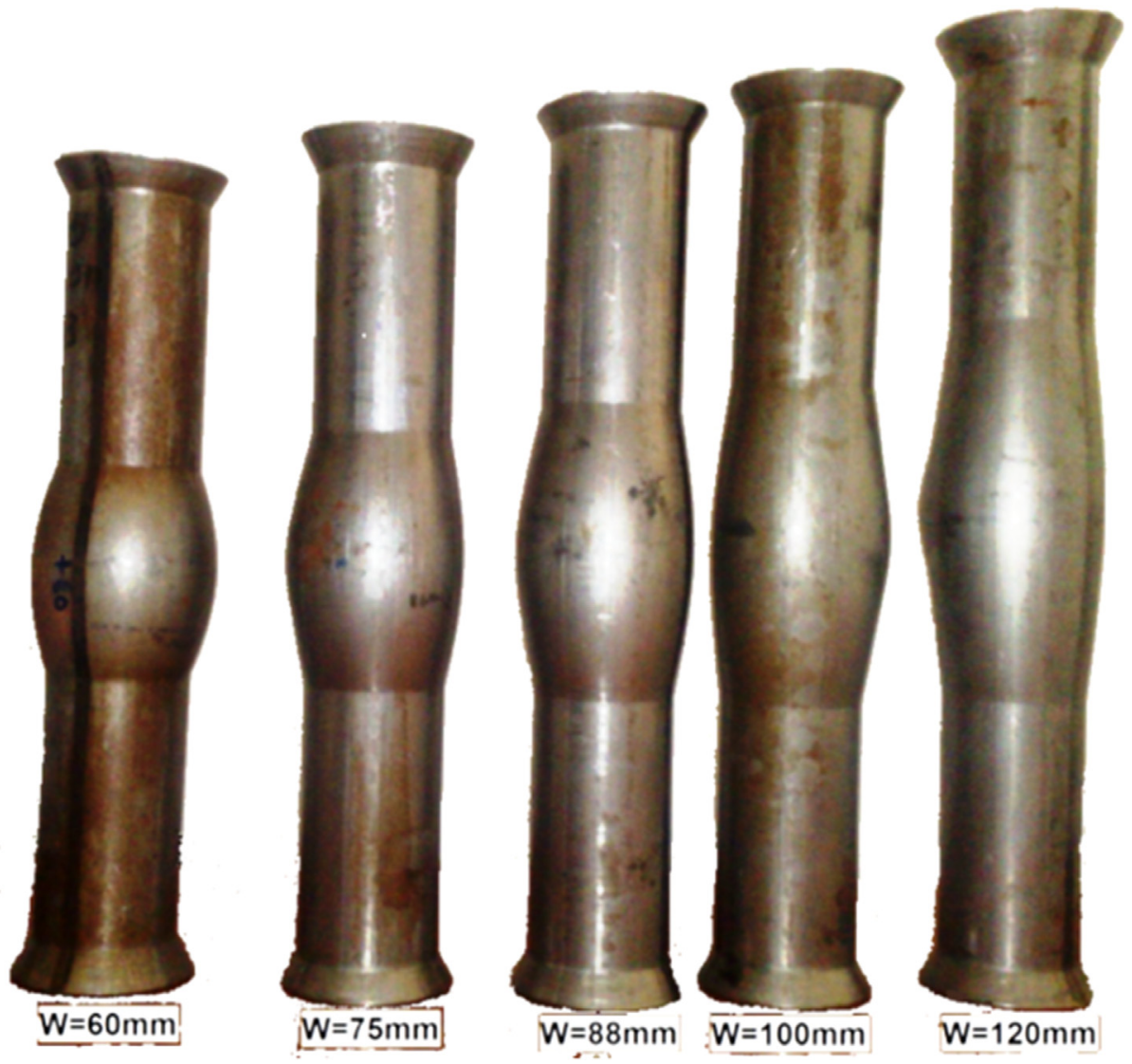

Fig. 2. Free hydroformed parts for different bulged widths.

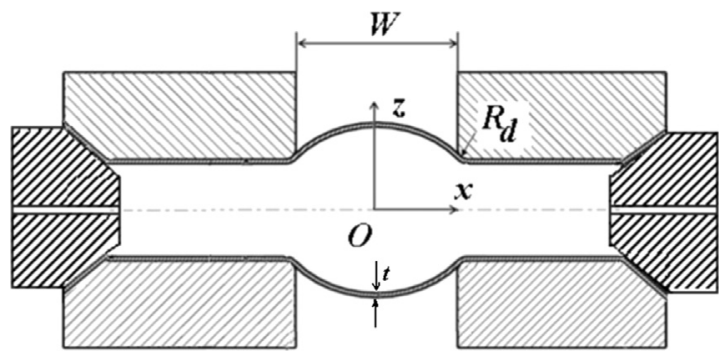

Fig. 3. Schematic diagram of free bulge test with clamped tube-ends.

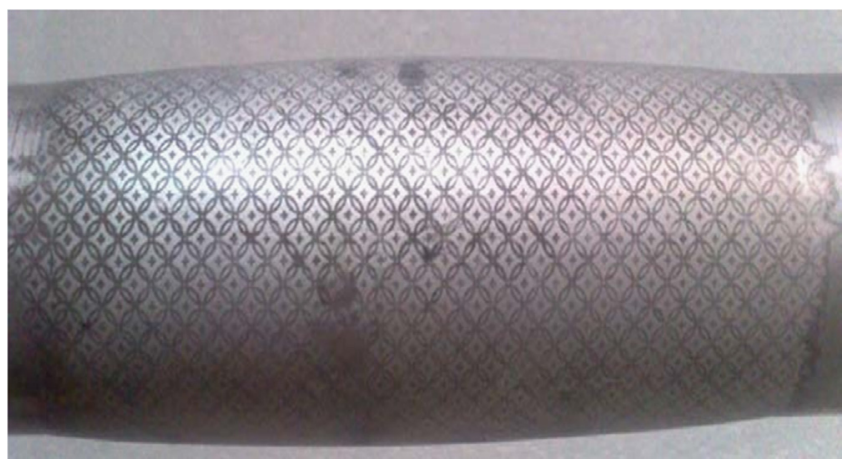

Fig. 4. Grid patterns etched onto tube's surface for experimental measurement of surface strains.

A grid with interlocking circular pattern was initially marked on the outside surface of the tube by electrolytically etching technique, as shown in Fig. 4. Applied grid pattern on tube external surface is measured before and after tube bulging in order to calculate hoop strain. Digital photos in the area of interest (e.g. the pole dome) are taken before and after deformation along the tube circumferential direction. The ratio of the pixels between photographs of the deformed and undeformed grid pattern along major allows calculating the hoop strain using digital image processing software. The strain measurement results are with the errors less than $1 \%$. The wall thickness at the pole dome was measured "offline" for each bulged tube at a given pressure using digital micron dial indicator gauge mounted on a self-designed apparatus [28]. The experimental wall thickness uncertainty at the pole was evaluated at $0.01 \mathrm{~mm}$. The bulge profile was also measured along the axial direction of the tube for different pressure levels. The different measured pressures and the pertaining bulge height, hoop strain, and thickness distribution at the pole are listed in Tables 3 and 4. The measured average value of the axial strain is about $0.002 \%$, thereby, it is too small compared to the hoop strain owing to the fixed tube ends during the free bulge test. The "offline" experimental measurements were repeated many times and performed on several bulged tubes. The recorded bulge profile, bulge height and thickness distribution are averaged across the tested tubes. Such procedure provides concise data, which represents the nominal performances of the tubular materials. It is worth mentioning that the experimental uncertainty on pressure measurements achieved "off-line" is about $0.2 \%$ for S235 hydroformed tubes and for the AA6063 bulged tubes, the discrepancy is less than $5 \%$. However, evaluated uncertainties remain as acceptable errors for an "off-line" measuring method.

\section{Constitutive modelling equations}

The elasto-plastic constitutive model considering isotropic elasticity and orthotropic plasticity is completely defined by 
Table 3

Experimental data obtained by the so-called stepwise bulge tests, evaluated at several pressure levels, in case of tube made of low carbon steel S235.

\begin{tabular}{lllll}
\hline $\begin{array}{l}\text { Level of } \\
\text { pressure }\end{array}$ & $\begin{array}{l}\text { Pressure } \\
(\mathrm{MPa})\end{array}$ & $\begin{array}{l}\text { Bulge height } \\
(\mathrm{mm})\end{array}$ & $\begin{array}{l}\text { Hoop strain } \\
(-)\end{array}$ & Thickness (mm) \\
\hline 1 & 19.440 & 0.43 & 0.018 & 1.05 \\
2 & 20.327 & 0.91 & 0.030 & 1.04 \\
3 & 20.923 & 1.52 & 0.050 & 1.01 \\
4 & 21.430 & 2.14 & 0.080 & 0.99 \\
5 & 21.695 & 2.56 & 0.093 & 0.97 \\
6 & 21.955 & 3.04 & 0.114 & 0.95 \\
7 & 22.023 & 3.20 & 0.119 & 0.94 \\
8 & 22.254 & 4.00 & 0.138 & 0.90 \\
9 & 22.360 & 4.88 & 0.157 & 0.88 \\
\hline
\end{tabular}

Table 4

Experimental data obtained by the so-called stepwise bulge tests, evaluated at several pressure levels, in case of tube made of AA6063 aluminium alloy.

\begin{tabular}{lllll}
\hline $\begin{array}{l}\text { Level of } \\
\text { pressure }\end{array}$ & $\begin{array}{l}\text { Pressure } \\
(\mathrm{MPa})\end{array}$ & $\begin{array}{l}\text { Bulge height } \\
(\mathrm{mm})\end{array}$ & $\begin{array}{l}\text { Hoop strain } \\
(-)\end{array}$ & Thickness (mm) \\
\hline 1 & 7.74 & 0.59 & 0.022 & 2.00 \\
2 & 8.74 & 1.21 & 0.045 & 1.95 \\
3 & 9.04 & 1.67 & 0.06 & 1.92 \\
4 & 9.25 & 2.14 & 0.085 & 1.88 \\
5 & 9.31 & 2.33 & 0.094 & 1.87 \\
6 & 9.38 & 2.65 & 0.101 & 1.84 \\
7 & 9.44 & 3.045 & 0.118 & 1.82 \\
8 & 9.60 & 3.98 & 0.135 & 1.77 \\
\hline
\end{tabular}

isotropic work-hardening law, a yield criterion and an associated flow rule. The yield criteria under analysis are Hill48 [32], Yld91 [33] and CB2001 [34].

The yield function is written in the following form:

$f\left(\underline{\boldsymbol{\sigma}}, \bar{\varepsilon}^{p}\right)=\bar{\sigma}(\underline{\boldsymbol{\sigma}})-Y\left(\bar{\varepsilon}^{p}\right)=0$

where $\bar{\sigma}$ is the equivalent stress, $\boldsymbol{\sigma}$ is the Cauchy stress tensor, $Y$ is the flow stress and $\bar{\varepsilon}^{p}$ is the equivalent plastic strain. It is assumed that $f$ is a convex function of $\boldsymbol{\sigma}$, and a positive homogeneous function of degree one. The plastic strain rate tensor can be determined from the associated flow rule:

$\underline{\dot{\varepsilon}}^{p}=\dot{\lambda} \frac{\partial f}{\partial \boldsymbol{\sigma}}$

where $\dot{\boldsymbol{\varepsilon}}^{p}$ is the plastic strain rate tensor and $\dot{\lambda}$ is the plastic multiplier. Because $f$ is a homogeneous function of degree one, $\dot{\bar{\varepsilon}}^{p}=\dot{\lambda}$. In a hypoelastic formulation, the plastic loading condition can be defined as follows:

$\dot{f}=0$, with $\quad \dot{\epsilon}^{p}=\dot{\lambda}>0$ and $\quad \ddot{\lambda} \dot{f}=0$.

\subsection{Isotropic work-hardening}

Assuming an isotropic strain hardening, two strain-hardening laws were chosen, namely Swift and Voce laws. The Swift law is typically adopted to model the isotropic hardening of steels, in which the flow stress $Y$ is given by

$Y=K\left(\varepsilon_{0}+\bar{\varepsilon}_{p}\right)^{n}$

where $K, \varepsilon_{0}$ and $n$ are the Swift's material parameters, and the initial yield stress, $Y_{0}$, is simply defined as $Y_{0}=K\left(\varepsilon_{0}\right)^{n}$. On the other hand, Voce law is usually preferred to describe the flow stress of materials exhibiting some saturation, as is the case of aluminium alloys. The Voce law is given by the expression

$Y=\sigma_{Y}+\left(\sigma_{s a t}-\sigma_{Y}\right)\left(1-\exp \left(-C_{y} \bar{\varepsilon}_{p}\right)\right)$
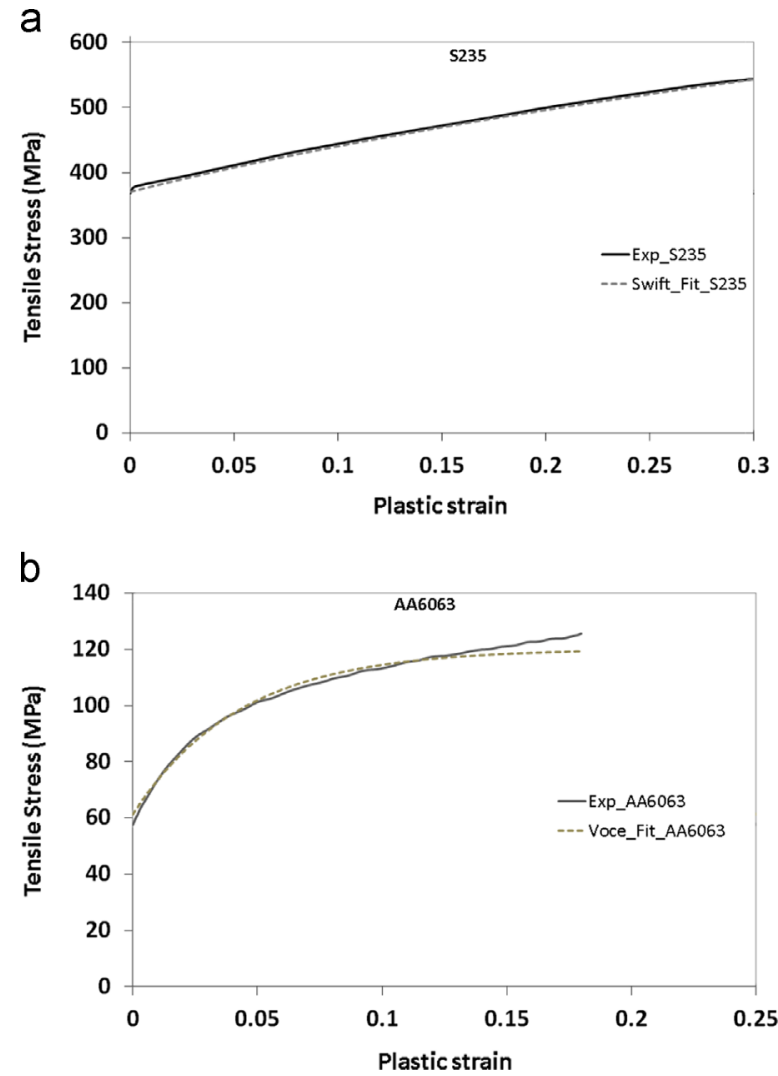

Fig. 5. True stress-strain curves obtained from uniaxial tensile tests in the reference direction along with fitting curves: (a) Swift law, in case of the S235 mild steel and (b) Voce law, in case of the AA6063 aluminium alloy.

Table 5

Work-hardening parameters: Swift and Voce functions.

\begin{tabular}{lrrrrr}
\hline \multicolumn{2}{l}{ Low carbon steel S235 } & \multicolumn{4}{c}{ Aluminium alloy AA6063 } \\
$\sigma_{y}(\mathrm{MPa})$ & $K(\mathrm{MPa})$ & $n$ & $\sigma_{y}(\mathrm{MPa})$ & $\sigma_{\text {sat }}(\mathrm{MPa})$ & $C_{y}$ \\
\hline 367.0 & 722.7 & 0.384 & 61.0 & 120.15 & 23.4 \\
\hline
\end{tabular}

where $\sigma_{y}$ is the initial yield stress, $\sigma_{\text {sat }}$ is the saturated flow stress and $C_{y}$ is the rate of saturation.

Fig. 5 shows the true stress-plastic strain curves obtained for both materials along with the best fitting curves obtained for Swift and Voce laws, for the steel and the aluminium alloy, respectively. Table 5 depicts the isotropic hardening material parameters for both Swift and Voce laws, determined by fitting the experimental tensile tests in the axial direction.

\subsection{Hill'48 yield criterion}

The Hill' 48 yield criterion [32] is an extension of the von Mises isotropic yield criterion to orthotropy. This yield criterion, generally used to describe the orthotropic behaviour of conventional rolled steel sheets, is defined as follows:

$$
\begin{gathered}
F\left(\sigma_{22}-\sigma_{33}\right)^{2}+G\left(\sigma_{33}-\sigma_{11}\right)^{2}+H\left(\sigma_{11}-\sigma_{22}\right)^{2}+2 L \sigma_{23}^{2} \\
+2 M \sigma_{31}^{2}+2 N \sigma_{12}^{2}=\bar{\sigma}^{2}
\end{gathered}
$$

where $F, G, H, L, M$ and $N$ are the anisotropy parameters; in case of sheets, the out of plane shear parameters, i.e. $L$ and $M$, are usually set equal to its isotropic value, $L=M=1.5$. The Hill48 yield criterion reduces to the isotropic von Mises when the $F, G, H$ are equal to 0.5 and $L, M$ and $N$ is set equal to 1.5 . 


\subsection{Yld91 yield criterion}

The Barlat yield criterion (Yld91) [33] is an extension of the non-quadratic isotropic yield criteria previously defined by Hershy [35] and Hosford [36]. The development of Yld91 is based on a linear transformation applied to the Cauchy stress tensor to compute the component of the isotropic plastic equivalent stress deviatoric tensor. The expression of Yld91 is written as follows:

$\left|S_{1}-S_{2}\right|^{m}+\left|S_{1}-S_{3}\right|^{m}+\left|S_{2}-S_{3}\right|^{m}=2 \bar{\sigma}^{m}$

where $S_{1}, S_{2}$ and $S_{3}$ are the principal values of the isotropic equivalent stress tensor $S$, obtained using a linear operator (a fourth-order tensor), which operates on the Cauchy stress tensor $\underline{\sigma}$ acting on the material, as follows:

$\underline{S}=\underline{L}: \underline{\sigma}$

The linear transformer tensor is defined for orthotropy as follows:

$\underline{L}=\left[\begin{array}{cccccc}\frac{c_{2}+c_{3}}{3} & -\frac{c_{3}}{3} & -\frac{c_{2}}{3} & 0 & 0 & 0 \\ -\frac{c_{3}}{3} & \frac{c_{3}+c_{1}}{3} & -\frac{c_{1}}{3} & 0 & 0 & 0 \\ -\frac{c_{2}}{3} & -\frac{c_{1}}{3} & \frac{c_{1}+c_{2}}{3} & 0 & 0 & 0 \\ 0 & 0 & 0 & c_{4} & 0 & 0 \\ 0 & 0 & 0 & 0 & c_{5} & 0 \\ 0 & 0 & 0 & 0 & 0 & c_{6}\end{array}\right]$

where $c_{1}, c_{2}, c_{3}, c_{4}, c_{5}$ and $c_{6}$ are the anisotropic coefficients of the yield criterion.

For sheet materials, the parameters $c_{4}$ and $c_{5}$ cannot be easily identified, and thus they are assumed to be equal to 1.0 which is their isotropic value. Thus, the anisotropic parameters of Yld91 yield locus are $c_{1}, c_{2}, c_{3}, c_{6}$ and the exponent $m$ which controls the shape of the yield surface. The $m$ coefficient is related to the materials crystallographic structure, being equal to 6 for BCC and equal to 8 for FCC materials [37].

\subsection{CB2001 yield criterion}

The Cazacu and Barlat (CB2001) [34] yield criterion is an extension of the Drucker's isotropic yield criterion to anisotropy [38]. Its formulation is based on the generalization of the second and third invariants of the deviatoric Cauchy stress tensor to orthotropy, being the yield function of CB2001 written as follows:

$\left(J_{2}^{0}\right)^{3}-c\left(J_{3}^{0}\right)^{2}=27\left(\frac{\bar{\sigma}}{3}\right)^{6}$

The second and third generalized invariants of the effective stress tensor are given by

$J_{2}^{0}=\frac{a_{1}}{6}\left(\sigma_{11}-\sigma_{22}\right)^{2}+\frac{a_{2}}{6}\left(\sigma_{22}-\sigma_{33}\right)^{2}+\frac{a_{3}}{6}\left(\sigma_{11}-\sigma_{33}\right)^{2}+a_{4} \sigma_{12}^{2}+a_{5} \sigma_{13}^{2}+a_{6} \sigma_{23}^{2}$

$$
\begin{aligned}
J_{3}^{0}=\frac{1}{27} & \left(b_{1}+b_{2}\right) \sigma_{11}^{3}+\frac{1}{27}\left(b_{3}+b_{4}\right) \sigma_{22}^{3}+\frac{1}{27}\left(2\left(b_{1}+b_{4}\right)-b_{2}-b_{3}\right) \sigma_{33}^{3} \\
& +\frac{2}{9}\left(b_{1}+b_{4}\right) \sigma_{11} \sigma_{22} \sigma_{33}-\frac{1}{3}\left(2 b_{9} \sigma_{22}-b_{8} \sigma_{33}-\left(2 b_{9}-b_{8}\right) \sigma_{11}\right) \sigma_{13}^{2} \\
& -\frac{1}{3}\left(2 b_{10} \sigma_{33}-b_{5} \sigma_{22}-\left(2 b_{10}-b_{5}\right) \sigma_{11}\right) \sigma_{12}^{2} \\
& -\frac{1}{3}\left(\left(b_{6}+b_{7}\right) \sigma_{11}-b_{6} \sigma_{22}-b_{7} \sigma_{33}\right) \sigma_{23}^{2}+2 b_{11} \sigma_{12} \sigma_{23} \sigma_{13}
\end{aligned}
$$

The anisotropy coefficients of the CB2001 yield criterion are 18: $a_{k}(k=1, \ldots, 6), b_{k}(k=1, \ldots, 11)$ and $c$. In case of $a_{k}=1.0$ and $b_{k}=1.0$ the criterion reduces to the isotropic Drucker's yield criterion. In case of sheets, and eliminating the out of plane shear related anisotropy parameters, the 18 anisotropy parameters reduces to only 11 , namely $a_{k}(k=1, \ldots, 4), b_{k}(k=1,2,3,4,5,10)$ and $c$.

\section{Parameter identification procedure}

The biaxial stretching is the most relevant strain path in the deformation process of tube hydroforming. However, the materials' experimental characterization is usually carried out from several uniaxial tensile tests, what is expected to be insufficient for an accurate calibration of constitutive parameters, given that the shape of the yield surfaces in the vicinity of biaxial stress states is not taken into account. So, to overcome this drawback, the free bulge test shall also be used as an additional experimental point to enhance the accuracy of yield surface around biaxial stress states. Thus, the challenge is to be able to determine the biaxial stress state at the pole of the bulged tube, in order to use the experimental information in the identification of the yield criteria parameters.

\subsection{Determination of biaxial stresses}

It is well-known that the stress state at the pole of the dome of the free bulge test is not an equibiaxial stress state but simply a biaxial stress state, being defined by the two unknown stresses $\sigma_{\theta}$ and $\sigma_{x}$, which define the normal stress components along the circumferential and axial (i.e. referential) directions, respectively. These values of stress components at the pole of the dome are estimated from FE analysis of the free bulge test with immovable tube's extremities. The parameters of stress-strain relationship are derived from the experimental pressure vs. bulge height response curve, commonly using the membrane theory and analytical model $[8,21]$. The resulting flow stress curve is used as input data to carry out the FE analysis of the free tube bulge test. Under an applied internal pressure $p i$, the equilibrium of a material element at the pole of the dome can be written as follows:

$$
\left(\frac{\sigma_{\theta}}{\rho_{\theta}}+\frac{\sigma_{x}}{\rho_{x}}\right)=\frac{p_{i}}{t}
$$

where $\rho_{\theta}$ and $\rho_{x}$ are the circumferential and axial radii of curvature; $p i$ and $t$ are the instantaneous internal pressure and tube wall thickness, respectively.

The axial profiles and wall thicknesses at the top of the dome, as well applied pressures are recorded for the stepwise bulge tests. The bulge profiles can be approximated using a second order polynomial equation, which is expressed as follows:

$z(x)=a_{0}+a_{1} x+a_{2} x^{2}$

where $z$ and $x$ are the pole point coordinates corresponding to the radial and axial directions and $a_{0}, a_{1}, a_{2}$ are the fitting polynomial coefficients. A second order polynomial equation was sufficient to reproduce the tube bulge profiles geometries at the top of the dome for the different applied pressures. Thus, for each bulge profile, a set of polynomial coefficients $\left(a_{0}, a_{1}, a_{2}\right)$ was determined by least-squares fitting method. This allows determining the radii of curvature $\rho_{\theta}$ and $\rho_{x}$ at the pole of the dome as follows:

$\rho_{\theta}=z_{\max }=\left|\frac{\left(4 a_{0} a_{2}-a_{1}^{2}\right)}{4 a_{2}}\right|$

$\rho_{x}=\left|\frac{\left[1+\left(z^{\prime}(x)^{2}\right)\right]^{3 / 2}}{z^{\prime \prime}(x)}\right|_{x=\left(-a 1 / 2 a_{2}\right)}$

where $z^{\prime}(x)$ and $z^{\prime \prime}(x)$ are the first and second derivatives of the current bulge height profile $z$ vs. the axial coordinate $x$. More details and insights into this well-known method for determining flow stress curve from free tube bulge test are given in Refs. $[8,20,21]$. 


\subsection{Artificial data generation}

The number of experimental points to be used in the identification procedure shall not be smaller than the number of parameters to be identified. In general, the experimental input data used for the identification of the yield criteria parameters encloses three directional yield stresses $\left(\sigma_{0^{\circ}}, \sigma_{45^{\circ}}, \sigma_{90^{\circ}}\right)$ and three $r$-values $\left(\mathrm{r}_{0^{\circ}}, \mathrm{r}_{45^{\circ}}, \mathrm{r}_{90^{\circ}}\right)$. This experimental information is usually enough to calibrate either Hill48 or Yld91 yield loci. However, for instance, to calibrate CB2001 yield criterion, in case of plane stress state, the necessary conventional input data includes $7 r$-values and 7 uniaxial yield stresses [39]. Furthermore, for accurate identification procedure of advanced yield functions [34,40,41], the required set of experimental input data includes, besides the seven uniaxial yield stresses and $r$-values, the equibiaxial yield stress $\sigma_{b}$, equibiaxial strain ratio $r_{b}$-value, and plane strain stresses [42,43], among others, such as biaxial stresses acquired from free expansion bulged tubes, which are needed for accurate calibration of constitutive models applied to simulate tube hydroforming process [44]. Nevertheless, the type of nonstandard experimental tests are usually not available in industrial or research laboratories, besides, it is uneconomical task to carry out a large number of costly mechanical tests.

The alternative proposal adopted here consists on the generation of some additional experimental data, somehow artificial but meaningful, at the aim to substitute the missing experimental results and to complete the required input data used for advanced yield criteria calibration. So, given that both Hill48 and Yld91 yield criteria anisotropy coefficients can be identified using only the three directional yield stresses and/or $r$-values, one is able to generate from these yield surfaces some additional data used as experimental points. The parameter identification of CB2001 yield function is considered herein as application to make the proof of such concept. Similar procedure has been followed by Khalfallah et al. [45] and applied for the parameter identification of anisotropic sheet metals, where the merits of producing and using artificial experimental points has been shown as a useful approach to complete an insufficient set of experimental data and leads to satisfactory findings.

In the present work, the identification of the anisotropy parameters of the yield criteria is based on the minimization of a costfunction using DD3MAT code [46], computer software specifically developed for this purpose. The cost-function is defined as follows:

$$
\begin{aligned}
\mathrm{F}_{o b j}(X)= & \sum_{i} w_{\mathrm{i}}\left(\frac{\sigma_{\psi}^{c a l}}{\sigma_{\psi}^{\text {exp }}}-1\right)^{2}+\sum_{i} w_{\mathrm{i}}\left(\frac{r_{\psi}^{\text {cal }}}{r_{\psi}^{\text {exp }}}-1\right)^{2} \\
& +w_{\mathrm{i}}\left(\frac{r_{b}^{\text {cal }}}{r_{b}^{\text {exp }}}-1\right)^{2}+w_{\mathrm{i}}\left(\frac{\sigma_{x}^{\text {cal }}}{\sigma_{x}^{\text {exp }}}-1\right)^{2}+w_{\mathrm{i}}\left(\frac{\sigma_{\theta}^{\text {cal }}}{\sigma_{\theta}^{\text {exp }}}-1\right)^{2}
\end{aligned}
$$

where $X$ is the set of anisotropy coefficients to be identified for a given yield criterion. $\sigma_{\psi}^{\exp }, r_{\psi}^{\exp }$ are either real of artificial experimental yield stresses and $r$-values, obtained from uniaxial tensile tests along the angle $\psi$ to the axial (or referential) direction; $\mathrm{r}_{b}^{\exp }$ is the artificial equibiaxial anisotropy ratio and $\sigma_{x}^{\exp }, \sigma_{\theta}^{\exp }$ are the experimental axial and circumferential stresses (see Section 4.1). $\sigma_{\psi}^{c a l}, r_{\psi}^{c a l}, r_{b}^{c a l}, \sigma_{x}^{c a l}$ and $\sigma_{\theta}^{c a l}$ are the reciprocal values predicted by the constitutive equations. $w_{i}$ are the weighting factors which can be adjusted and assigned differently to each experimental values. In present work, one has assumed that the weights related to real experimental data should be higher than the weights assigned to the so-called artificial data. For sake of simplicity, weighting factors for effective and artificial experimental input data were assumed to be of the order 1.0 and 0.1 , respectively.
Hill48 and Yld91 yield criteria were identified using the experimental data available, i.e., three directional yield stresses $\left(\sigma_{0^{\circ}}, \sigma_{45^{\circ}}, \sigma_{90^{\circ}}\right)$, three $r$-values $\left(\mathrm{r}_{0^{\circ}}, \mathrm{r}_{45^{\circ}}, \mathrm{r}_{90^{\circ}}\right)$ and the experimental axial and circumferential stresses. For both yield criteria the minimization of Eq. (17) corresponds to an over-constrained problem, since the number of anisotropy parameters is less than the number of experimental values. It is known that Hill48 and the Yld91 cannot simultaneously describe both directional yield stresses and $r$-values. In this context, it is important to mention that the use of a weighting factor equal for both the directional yield stresses and the $r$-values leads to solutions that tend to fit the $r$-values more accurately than yield stresses.

CB2001 yield criterion involves 11 anisotropy coefficients in the plane stress case, therefore, at least 11 experimental values are required for its calibration. The missing input data is the additional directional tensile stresses and $r$-values along with biaxial values, which are generated using the Yld91 yield criterion. Thus, the first step of the CB2001 identification procedure consists on the calibration of the Yld91 criterion using input data gathered from the three tensile tests carried out at orientations of $0^{\circ}, 45^{\circ}$ and $90^{\circ}$ to RD. Since Yld91 is not flexible enough to simultaneously accurately reproduce $r$-values and directional tensile stresses, two different combinations of the weighting factors are assigned to the experimental data. To determine the artificial yield stress values, high weighting factors are assigned to the experimental yield stresses to privilege their reproduction by the Yld91, while low weighting factors are attributed for the $r$-values. The calibration used to predict the $r$-values inverts the weighting factors. The values of 1.0 and 0.001 are used as high and low weighting factors, respectively.

\subsection{Low carbon steel S235}

The full set of effective experimental results used as input data for the identification of anisotropy parameters of all yield criteria, namely Hill48, Yld91 and CB2001, is listed in Table 6 for the S235 low carbon steel. For an equivalent plastic strain of $\bar{\varepsilon}^{\mathrm{pl}}=10 \%$, the biaxial stresses along the axial and circumferential directions are obtained from FE analysis which reproduces the experimental curve of the free tube bulge test for the steel S235. In Table 6, bold text refers to results obtained from effective experiments and normal text related to artificial data predicted using by Yld91 yield criterion. The anisotropy parameters determined for the Hill48, Yld91 and CB2001 yield criteria in case of the tubular material S235 are depicted in Table 7.

Figs. 6 and 7 show the plots of the profiles of anisotropy of both the $r$-values and the yield stresses along with real and artificial experimental data. The plotted yield stresses correspond to an equivalent plastic strain value of $10 \%$. The effective experimental results are represented by circular filled symbols and the artificial ones are assigned by squared filled symbols. One can observe that all the studied yield criteria predict similar evolutions of the $r$ values; however, in case of the yield stresses prediction, only

Table 6

Mechanical properties used as input data to calibrate the yield criteria for the low carbon steel S235 (bold: effective experimental data).

\begin{tabular}{llllll}
\hline $\begin{array}{l}\text { Angle } \\
\text { from RD }\end{array}$ & $r$-Value & $\begin{array}{l}\text { Uniaxial } \\
\text { stress at } \\
\bar{\varepsilon}^{\mathrm{pl}}=10 \%\end{array}$ & $\begin{array}{l}\sigma_{x}(\mathrm{MPa}) \\
\bar{\varepsilon}^{\mathrm{pl}}=10 \%\end{array}$ & $\begin{array}{l}\sigma_{\theta}(\mathrm{MPa}) \\
\bar{\varepsilon}^{\mathrm{pl}}=10 \%\end{array}$ & $\begin{array}{l}\text { Equibiaxial } r- \\
\text { value }\end{array}$ \\
\hline 0 & $\mathbf{1 . 4 8}$ & $\mathbf{4 4 2 . 9}$ & $\mathbf{3 0 1 . 3}$ & $\mathbf{5 6 0 . 4}$ & 0.98 \\
30 & 1.04 & 457.0 & & & \\
45 & $\mathbf{0 . 9 2}$ & $\mathbf{4 6 7 . 4}$ & & & \\
60 & 1.05 & 457.4 & & & \\
90 & $\mathbf{1 . 5 1}$ & $\mathbf{4 3 8 . 2}$ & & & \\
\hline
\end{tabular}


Table 7

Anisotropy parameters obtained for Hill48, Yld91 and CB2001 with experimental and artificial data for low carbon steel S232.

\begin{tabular}{lllllll}
\hline Hill48 & $\mathrm{F}$ & $\mathrm{G}$ & $\mathrm{H}$ & $\mathrm{N}$ & & \\
& 0.42237 & 0.43178 & 0.63341 & 1.2225 & & \\
Yld91 & $\mathrm{c}_{1}$ & $\mathrm{c}_{2}$ & $\mathrm{c}_{3}$ & $\mathrm{c}_{6}$ & $\mathrm{~m}$ & \\
& 0.92799 & 0.93592 & 1.08716 & 0.91451 & 6 & \\
CB2001 & $\mathrm{a}_{1}$ & $\mathrm{a}_{2}$ & $\mathrm{a}_{3}$ & $\mathrm{a}_{4}$ & $\mathrm{c}$ & \\
& 1.13118 & 1.001079 & 0.999766 & 0.870211 & 0.333811 & \\
& $\mathrm{~b}_{1}$ & $\mathrm{~b}_{2}$ & $\mathrm{~b}_{3}$ & $\mathrm{~b}_{4}$ & $\mathrm{~b}_{5}$ & $\mathrm{~b}_{10}$ \\
& 0.94411 & 3.237712 & 3.508755 & -0.565 & 0.301428 & 0.390908 \\
\hline
\end{tabular}

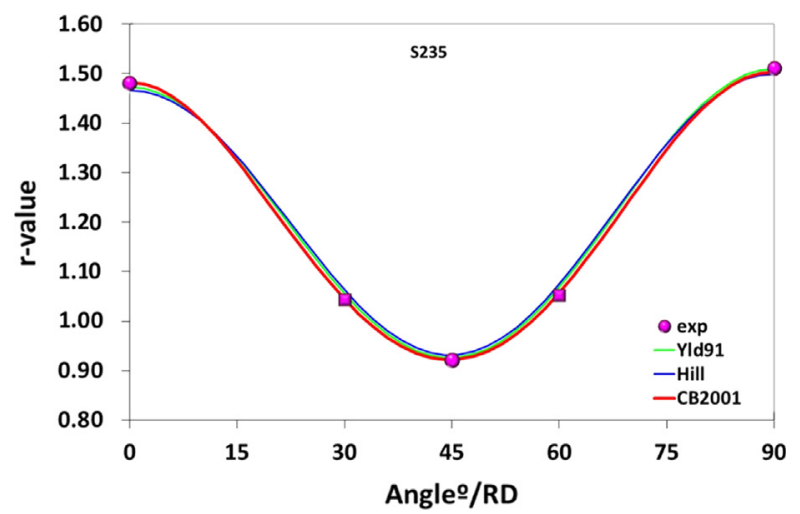

Fig. 6. Predicted $r$-values by Hill48, Yld 91 and CB2001 yield criteria along with experimental (circular dots) and artificial (square dots) data points for the S235 mild steel.

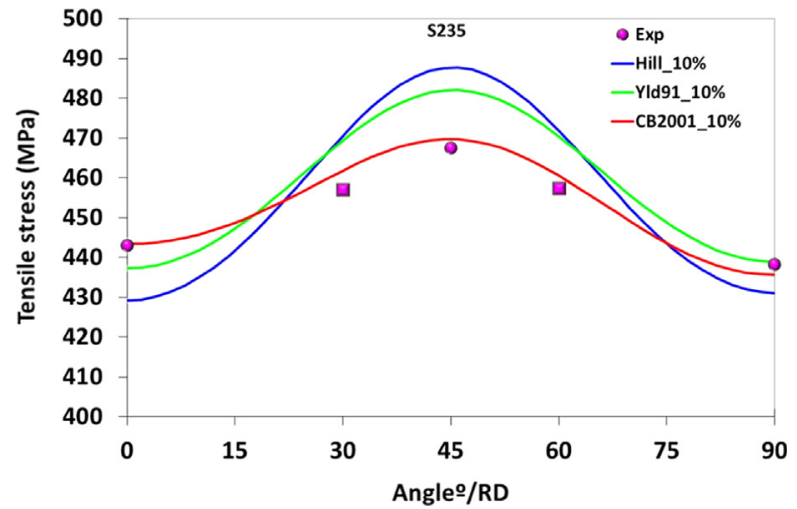

Fig. 7. Predicted tensile stresses by Hill48, Yld91 and CB2001 yield criteria along with experimental (circular dots) and artificial (square dots) data points for the S235 mild steel.

CB2001 is able to well describe with accuracy the experimental behaviour. Fig. 8 shows a comparison between the shapes of the yield surfaces determined by the constitutive equations and the experimental values (von Mises yield surface was added for comparison purposes). From the analysis of Fig. 8, one can also see that the biaxial stress state at the pole of the dome, i.e. defined by the stresses components (301.3 MPa, 560.4 MPa), is better described and accurately captured by the CB2001yield criterion. This is undeniably owing to the higher flexibility of CB2001 yield criterion, which is expressed by a large number of anisotropy parameters. Fig. 9 shows the CB2001 yield surfaces calculated for different levels of shear stress $\sigma_{12}$.

\subsection{Aluminium alloy AA6063}

The complete set of experimental results used as input data for the calibration of the yield criteria, namely Hill48, Yld91 and CB2001 is summarized in Table 8 for the aluminium alloy AA6063.

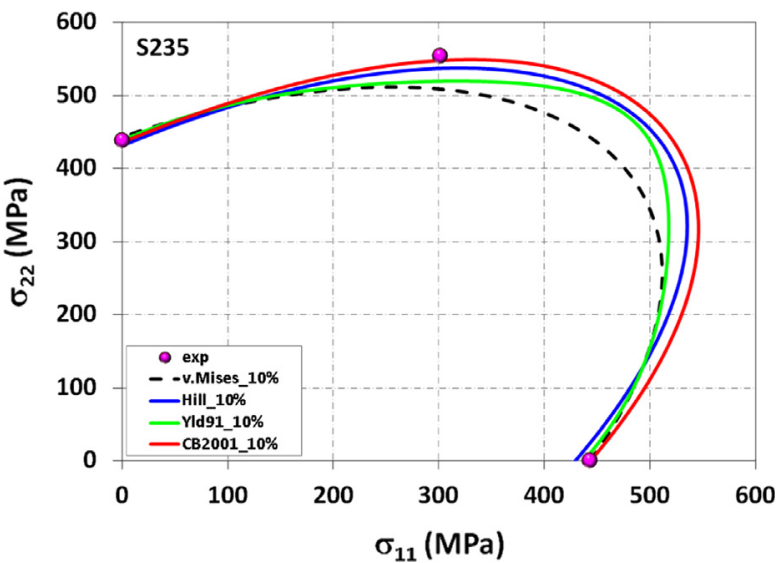

Fig. 8. Yield surface contours predicted by Mises, Hill48, Yld91 and CB2001 yield criteria for an equivalent plastic strain of $10 \%$ in the $\sigma_{11}$ vs. $\sigma_{22}$ space, in the case of the S235 mild steel.

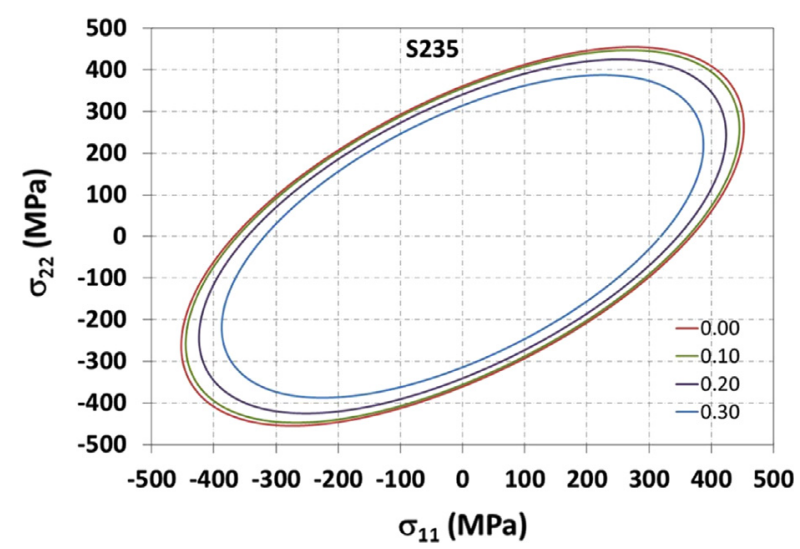

Fig. 9. Predicted initial yield surfaces, determined from the CB2001 yield criterion, plotted in the $\sigma_{11}$ vs. $\sigma_{22}$ space for different levels of the normalized shear stress $\sigma_{12}$, in case of S235 mild steel.

Table 9 reports the anisotropy parameters for all the studied yield criteria.

Figs. 10 and 11 show the evolution of the $r$-values and yield stresses along with the experimental results. The Hill48, Yld91 and CB2001 yield criteria predict roughly similar evolutions of the $r$ values. However, yield stresses anisotropy evolution is better predicted by the CB2001 yield criterion, demonstrating that it is capable to accurately describe the strongly anisotropic behaviour of the AA6063 aluminium alloy, where the other yield criteria fail to predict that experimental data.

Fig. 12 depicts von Mises, Hill48, Yld91 and CB2001 yield surfaces and experimental points plotted in the first quadrant of the yield locus. From Fig. 12, one can see clearly the large deviation between the results obtained using von Mises, Hill48, Yld91 yield criteria and the experimental points. Solely, CB2001 yield surface plot passes through experimental points and particularly, it is closer to the experimental biaxial state point defined by the stress components (62.0 MPa, 114.7 MPa), than the other yield surfaces (i.e., v.Mises, Hill48 and Yld91). Accordingly, the higher flexibility of the CB2001 yield criterion, owing to its wide number of anisotropy parameters, enables accurate description of strongly anisotropic material behaviour of aluminium alloys. Fig. 13 shows the yield surfaces predicted by the CB2001 yield criterion and plotted for different levels of shear stresses $\sigma_{12}$. 
Table 8

Mechanical properties used as input data to calibrate the yield criteria for the AA6063 (bold: effective experimental data).

\begin{tabular}{lcllll}
\hline $\begin{array}{l}\text { Angle } \\
\text { from RD }\end{array}$ & $r$-Value & $\begin{array}{l}\text { Uniaxial } \\
\text { stress at } \\
\bar{\varepsilon}^{\mathrm{pl}}=10 \%\end{array}$ & $\begin{array}{l}\sigma_{x}(\mathrm{MPa}) \\
\bar{\varepsilon}^{\mathrm{pl}}=10 \%\end{array}$ & $\begin{array}{l}\sigma_{\theta}(\mathrm{MPa}) \\
\bar{\varepsilon}^{\mathrm{pl}}=10 \%\end{array}$ & $\begin{array}{l}\text { Equibiaxial } r- \\
\text { value }\end{array}$ \\
\hline 0 & $\mathbf{0 . 5 9}$ & $\mathbf{1 1 2 . 8}$ & $\mathbf{6 2 . 0}$ & $\mathbf{1 1 4 . 7}$ & 0.78 \\
30 & 0.74 & 82.9 & & & \\
45 & $\mathbf{0 . 8 3}$ & $\mathbf{7 7 . 4}$ & & & \\
60 & 0.85 & 77.3 & & & \\
90 & $\mathbf{0 . 7 9}$ & $\mathbf{8 1 . 8}$ & & & \\
\hline
\end{tabular}

Table 9

Anisotropy parameters obtained for Hill48, Yld91 and CB2001 with experimental and artificial data for AA6063.

\begin{tabular}{lllllll}
\hline Hill48 & $\mathrm{F}$ & $\mathrm{G}$ & $\mathrm{H}$ & $\mathrm{N}$ & & \\
& 0.739914 & 0.946706 & 0.581560 & 2.266875 & & \\
Yld91 & $\mathrm{c}_{1}$ & $\mathrm{c}_{2}$ & $\mathrm{c}_{3}$ & $\mathrm{c}_{6}$ & $\mathrm{~m}$ & \\
& 1.174504 & 1.280609 & 1.100505 & 1.186848 & 8 & \\
CB2001 & $\mathrm{a}_{1}$ & $\mathrm{a}_{2}$ & $\mathrm{a}_{3}$ & $\mathrm{a}_{4}$ & $\mathrm{c}$ & \\
& 1.454720 & 1.958297 & -0.66596 & 1.781621 & -2.68147 & \\
& $\mathrm{~b}_{1}$ & $\mathrm{~b}_{2}$ & $\mathrm{~b}_{3}$ & $\mathrm{~b}_{4}$ & $\mathrm{~b}_{5}$ & $\mathrm{~b}_{10}$ \\
& 0.904973 & 2.327966 & 0.335891 & -1.29223 & 2.553535 & 3.278480 \\
\hline
\end{tabular}

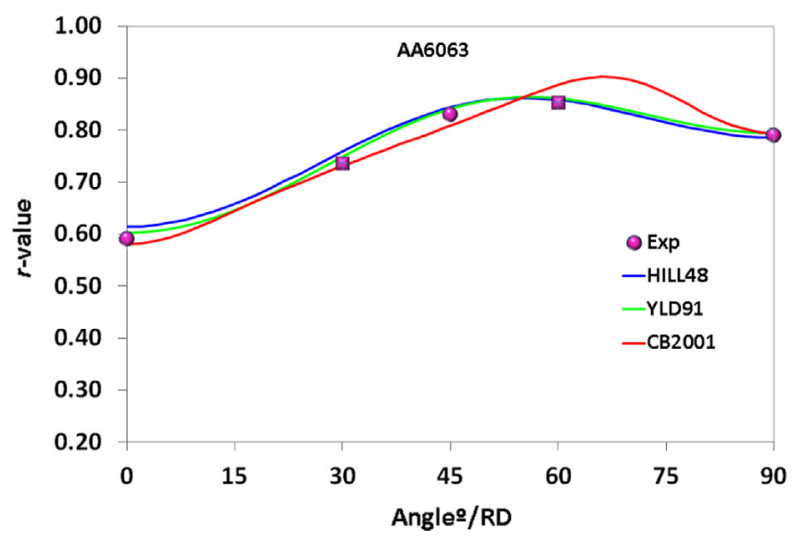

Fig. 10. Predicted $r$-values by Hill48, Yld91 and CB2001 yield criteria along with experimental (circular dots) and artificial (square dots) data points for the AA6063 aluminium alloy.

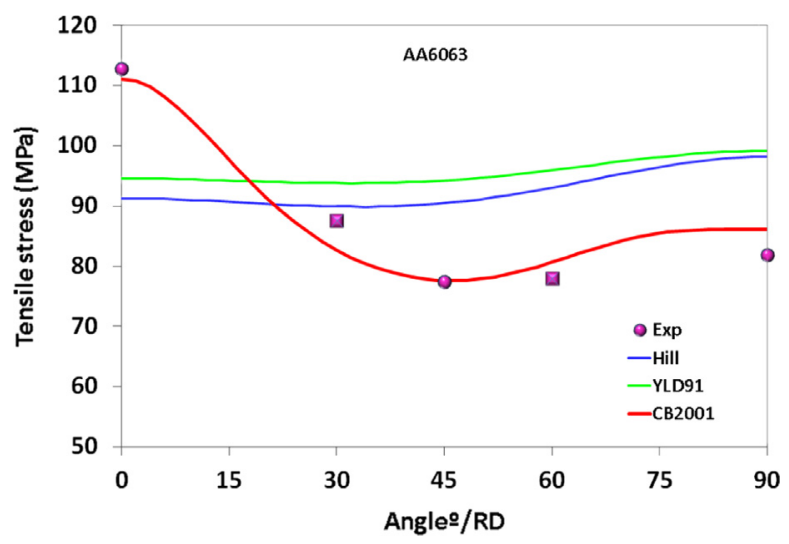

Fig. 11. Predicted tensile stresses by Hill48, Yld91 and CB2001 yield criteria along with experimental (circular dots) and artificial (square dots) data points for the AA6063 aluminium alloy.

\section{Numerical validation of the identification procedure}

In order to gain better insight into the accuracy of the adopted material parameter identification procedure, numerical simulations of

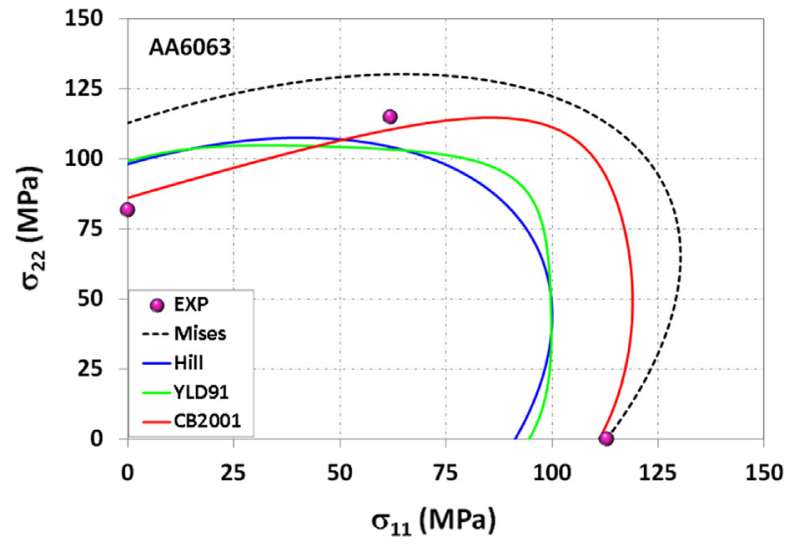

Fig. 12. Yield surfaces contours predicted by Mises, Hill48, Yld91 and CB2001 yield criteria for an equivalent plastic strain of $10 \%$ in the $\sigma_{1}$ vs. $\sigma_{2}$ space for the AA6063 aluminium alloy.

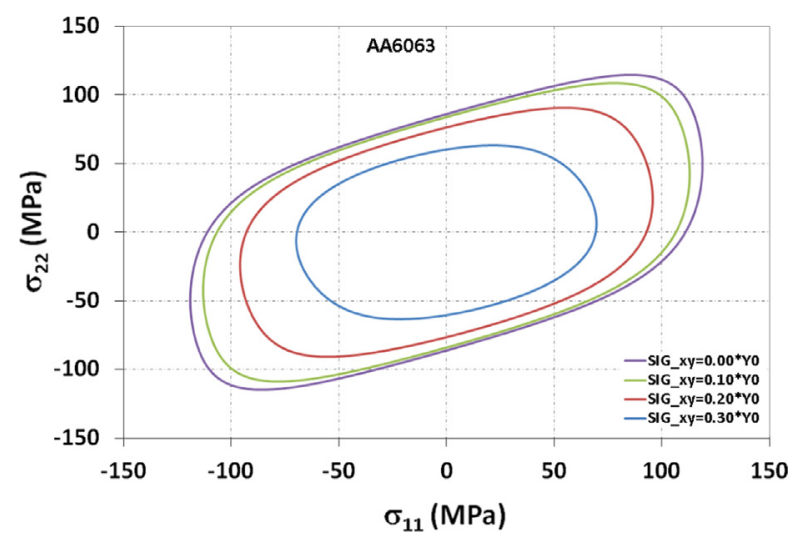

Fig. 13. Predicted initial yield surfaces, determined from the CB2001 yield criterion, plotted in the $\sigma_{11}$ vs. $\sigma_{22}$ space for different levels of the normalized shear stress $\sigma_{12}$, in case of AA6063 aluminium alloy.

the free bulge test are performed for the two materials. Basically, the purpose of these simulations is to validate the proposed identification procedure and to access the prediction accuracy of the studied constitutive equations. Finite element analysis was performed using the DD3IMP in-house finite element code [47]. Taking into account the geometric, loading, and material symmetries, one eighth of the whole part was modelled in order to reduce the computational time. The discretization of the tube combines two finite element sizes. The central bulging area is discretized with an element size of $1 \mathrm{~mm} \times 1.3 \mathrm{~mm}$ along the circumferential and axial directions, respectively. A coarse mesh is used to discretize the remaining part of the tube. Tri-linear 8-node hexahedral solid elements associated to a selective reduced integration (SRI) are used, with two layers of elements through-thickness. The free bulge test has been simulated taking into account the tool geometry, which includes the die for guiding the tube during deformation, retaining the tube's extremities and controlling the free bulged area. The tools are modelled as a rigid body. The die entrance radius is set to $7.5 \mathrm{~mm}$ and the free bulged width is $60 \mathrm{~mm}$. The tube external diameter is set to $50 \mathrm{~mm}$, the initial thickness is $1.07 \mathrm{~mm}$ for S235 and $2.04 \mathrm{~mm}$ for AA6063 aluminium alloy. A constant friction coefficient of 0.08 is assumed for all the contact interfaces. This value was estimated based on experimental conditions and on sensitivity analysis performed by FEA. The internal pressure was defined as a linear time-dependent curve. Fig. 14 shows the $3 \mathrm{D}$ finite element model used to simulate the free bulge test.

Fig. 15 shows the experimental free bulged part and the 3D numerical simulation of the test. Fig. 15(a) shows the tube's conical 


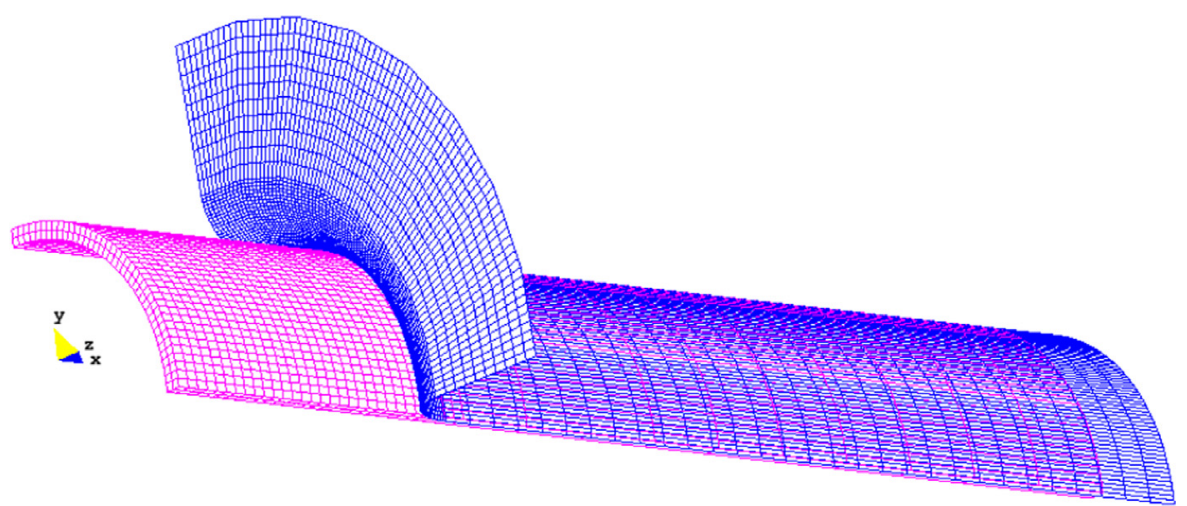

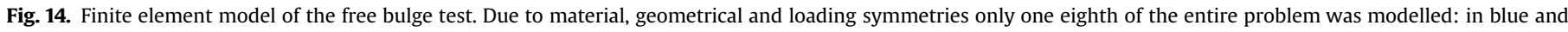

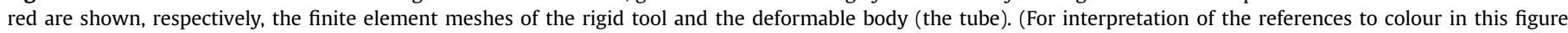
legend, the reader is referred to the web version of this article.)

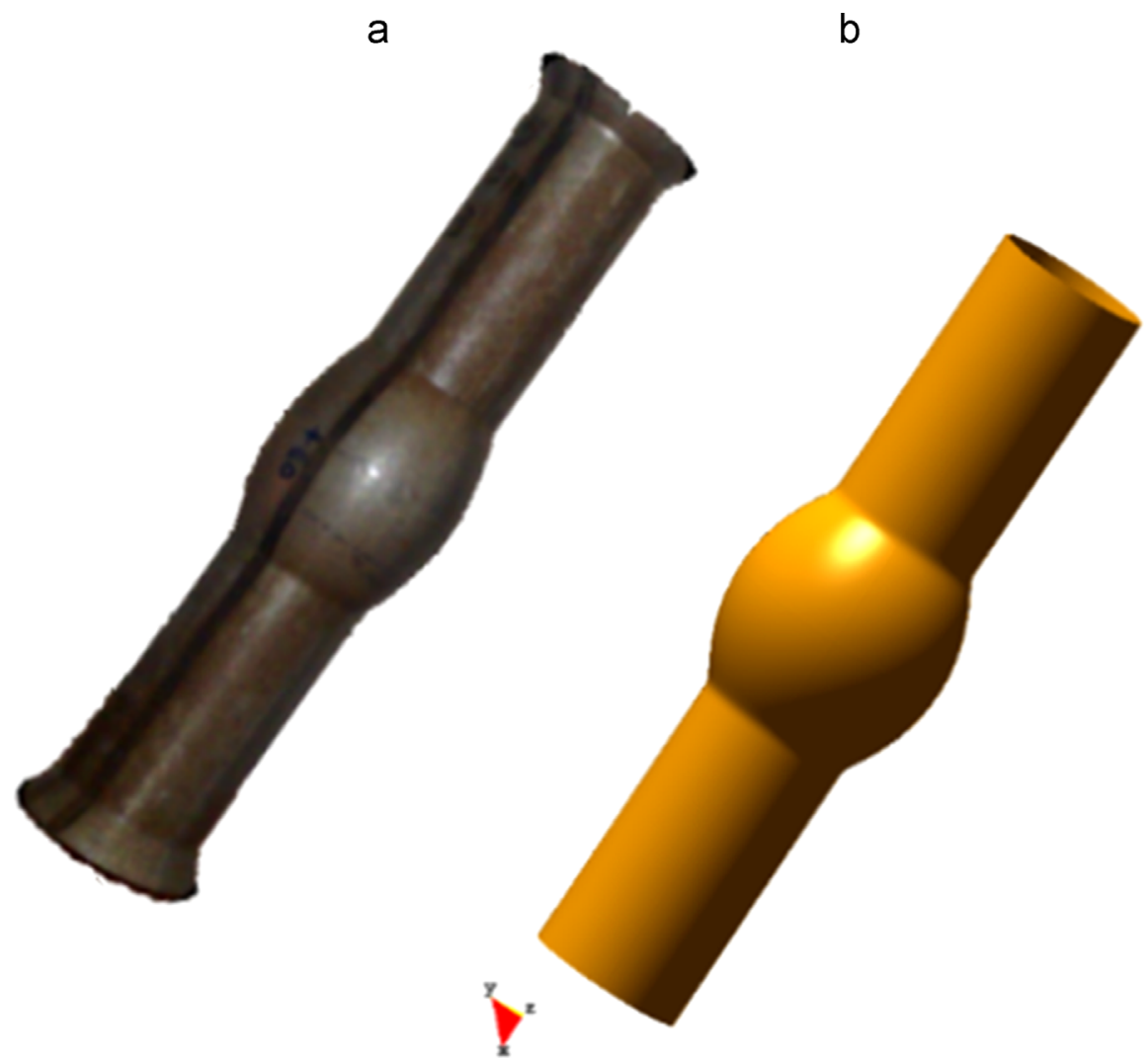

Fig. 15. Free bulge test: (a) experimental free bulged part and (b) simulation of free bulge test.

ends which are obtained at the beginning of test by crashing the tube extremities against a chamfer manufactured in the die inserts, so they lock the tube in the axial direction. However, for the FE model, (Fig. 15(b)), the boundary conditions which correspond to the tube ends are established by setting the degrees of freedom for all nodes equal to zero.

\subsection{Case of low carbon steel S235}

FE simulations of the free bulge test for S235 were carried out using the three anisotropic yield functions, Hill48, Yld91 and CB2001 and input data given in Tables 5 and 7. von Mises yield criterion is also included and considered as a reference for comparison purposes. Fig. 16 shows the comparison between numerical results of the bulge height plotted against the internal pressure, pertaining to the different yield criteria, along with the experimental results. This figure also depicts clearly that the bulge height vs. pressure curve predicted by the CB2001 yield criterion accurately fits the experimental points. Also, it shows the limited ability of von Mises, Hill48 and Yld91 yield criteria to accurately predict the stress path dictated by the free bulge test conditions, as previously shown in Fig. 8. Additionally, Fig. 16 emphasizes that the bulge height increases slowly at the first stage of pressure increments up to a certain level that characterizes the yield pressure. Starting from this point, the pole height increases rapidly within a narrow pressure range, until tube bursting. All the models predict a similar yield pressure, comparable to the experimental value, which is equal to $16.5 \mathrm{MPa}$. This pressure is generally related to the initial tube thickness, the tube outer diameter and the material initial yield stress [48]. The similarity between estimated yield pressures for the different models is confirmed by the yield surfaces plots presented in Fig. 8. Contrarily, predicted fracture 
pressures by yield criteria are in disagreement with the experimental value of $22.5 \mathrm{MPa}$, except for the one predicted by CB2001 and it is in agreement with the experimental findings.

To gain further insight into the effect of the adopted yield criterion on the deformation behaviour prediction of free hydroformed tubes, experimental pole thickness along with numerical results are compared. Fig. 17 shows a good agreement between predicted thickness distribution by CB2001 yield criterion and experimental results. Conversely, thickness distribution predicted using von Mises, Hill48 and Yld91 yield criteria underestimate the experimental thickness for a given internal pressure.

Fig. 18 shows the thickness evolution plots of the bulge at the pole against the bulge height. It is expected that the thickness distribution predicted by the different yield criteria present a clear distinction in the pole thickness, analogous to what was observed in Fig. 17. However, as shown in Fig. 18, the thickness evolves linearly with the bulge height for all yield criteria, in agreement with the experimental data. This demonstrates the almost linear relationship between the thickness and the bulge height when using free bulge test with locked tube extremities. Since the axial strain is negligible for such test, the strain through the tube thickness is roughly equal to the hoop strain used to evaluate the tube height at the pole.

Fig. 19 shows for some pressure levels, the experimental and numerical profiles predicted by CB2001 yield criterion. The profiles predicted by the other yield criteria are in disagreement with the experimental results. It is worth noticing that the die entrance radius has an effect in the prediction of the bulge profiles;

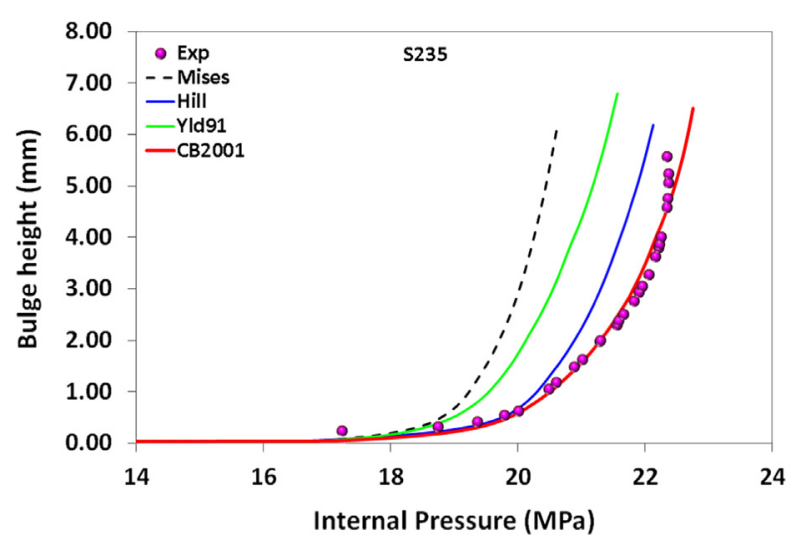

Fig. 16. Comparison between the bulge height vs. pressure curves determined experimentally and numerically predicted by all studied yield criteria, in case of S235 mild steel.

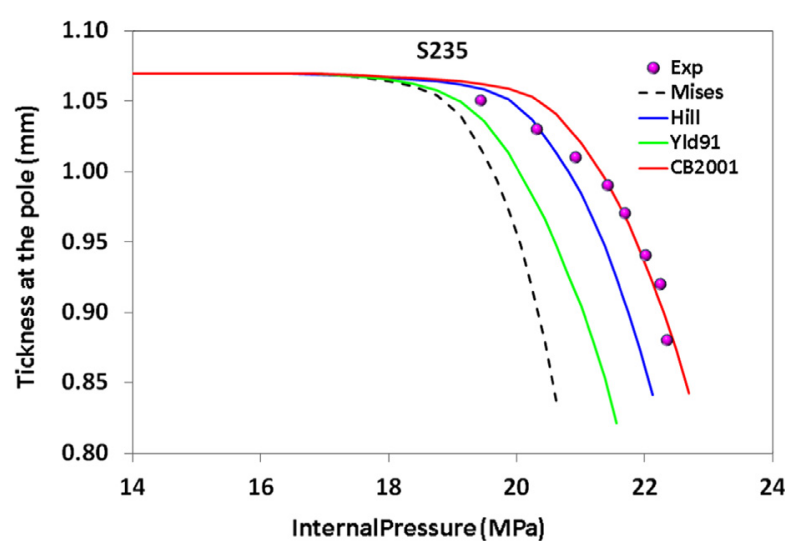

Fig. 17. Comparison between the evolutions of the thickness at the pole with the pressure evolution, as determined experimentally and numerically from all studied yield criteria, in case of S235 mild steel.

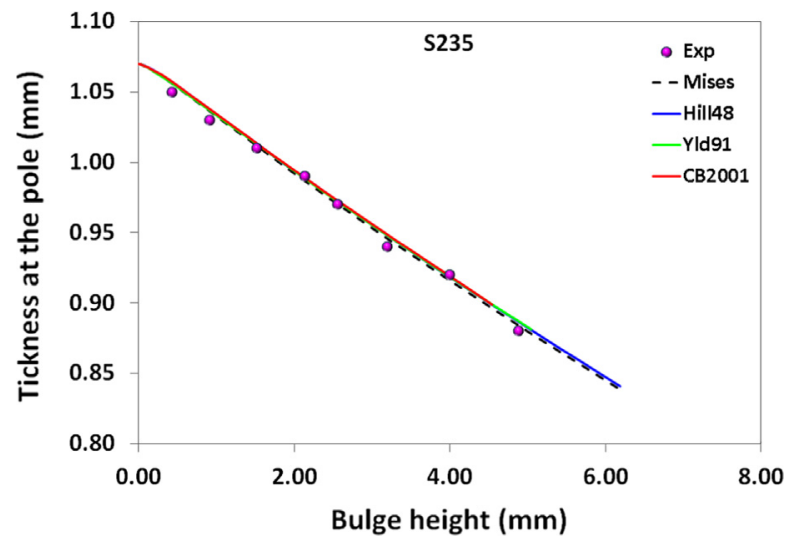

Fig. 18. Comparison between experimental and numerical thicknesses at the top of the pole vs. bulge height curves using the four yield criteria for S235 mild steel.

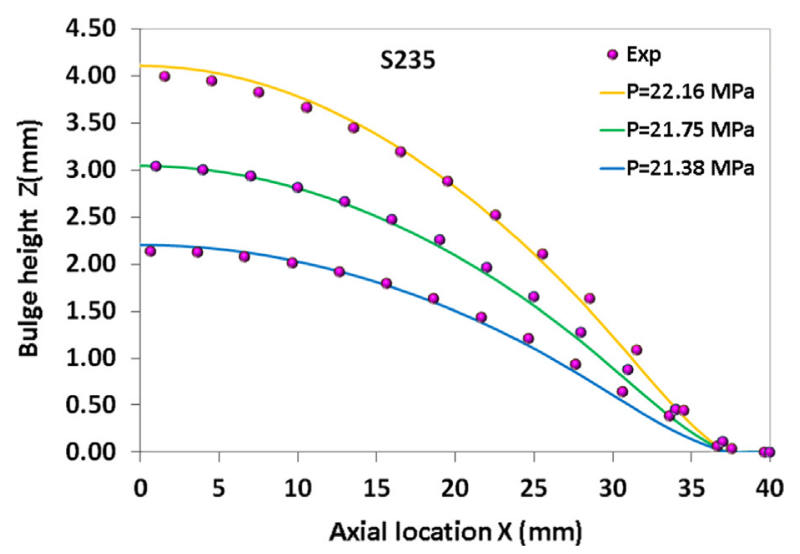

Fig. 19. Experimental and numerically predicted bulge profiles using the CB2001 yield criterion, for the S235 mild steel.

particularly in the regions close to the die radius. Thus, to improve the correlation between experimental and numerical profiles, the die radius should be accurately measured and modelled [28].

\subsection{Case of aluminium alloy AA6063}

The same methodology was followed for the aluminium alloy AA6063 as for the low carbon steel S235. The constitutive models' parameters are listed in Tables 5 and 9, and numerical results were obtained considering von Mises, Hill48, Yld91 and CB2001 yield criteria. Fig. 20 presents the predicted along with experimental results of bulge height $v$ s. pressure curves. It is observed that the response obtained using the CB2001 yield criterion is very close to the experimental data, particularly when compared to the other predictions. The internal pressures predicted using Hill48 and Yld91 yield criteria are similar. These models underestimate the experimental bulge height results, whilst the von Mises model overestimates them. The observed materials behaviour in Fig. 20 can be forecasted by the yield locus presented in Fig. 12 .

The yield surface plots show that only CB2001 yield criterion goes through the experimental point defining the biaxial stress state experienced by the tube at the pole dome. Thus, the CB2001 yield criterion is capable to accurately describe the AA6063 material behaviour of the free bulge test. Moreover, yield pressures predicted by the different yield functions are dissimilar. In fact, these pressures are $4.80 \mathrm{MPa}, 4.86 \mathrm{MPa}$ and $5.72 \mathrm{MPa}$, for Yld91, Hill48 and von Mises yield criteria, respectively. The CB2001 predicts a yield pressure equal to $4.88 \mathrm{MPa}$ close to the experimental value, which is evaluated at $4.50 \mathrm{MPa}$. This is not surprising since the yield pressure is related to the initial yield stress and the yield 


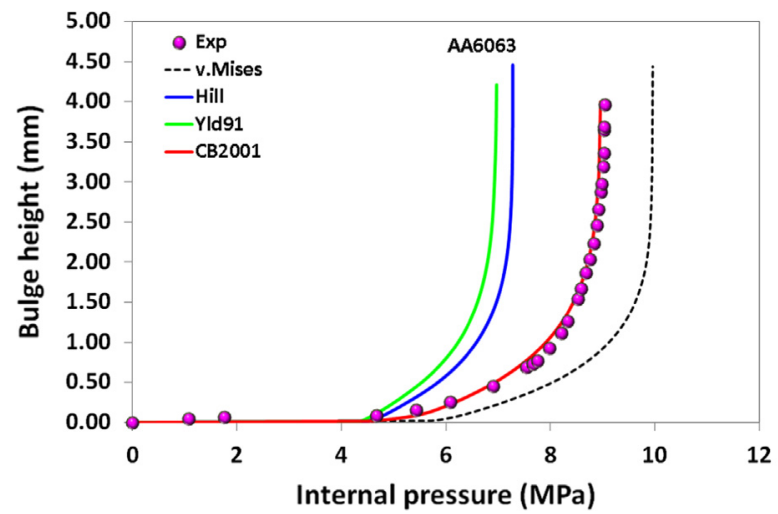

Fig. 20. Comparison between the Bulge height vs. Pressure curves determined experimentally and numerically predicted by all studied yield criteria, in case of AA6063 aluminium alloy.

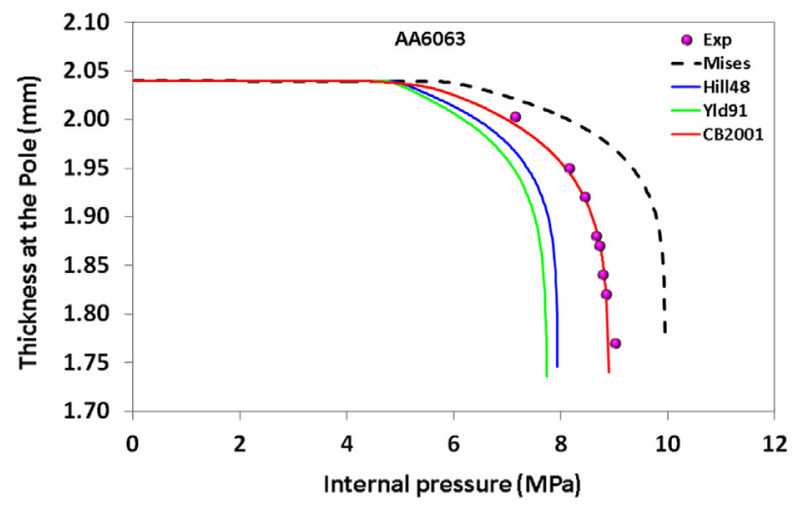

Fig. 21. Comparison between the evolutions of the thickness at the pole with the pressure evolution, as determined experimentally and numerically from all studied yield criteria, in case of AA6063 aluminium alloy.

criteria predict different initial yield stresses, as shown in Fig. 12. It is noteworthy that the plastic behaviour of the AA6063 is fairly dissimilar from the steel S235 behaviour and this is owing to the strongly marked anisotropy behaviour of the Aluminium alloy AA6063 compared to the steel S235.

Regarding the hardening behaviour of the AA6063, it is observed that beyond the yield pressure, the bulge height increases gradually with pressure evolution, until the bulge height reaches a value around $1.5 \mathrm{~mm}$. Starting from this value, the internal pressure slowly rises at a roughly constant rate, whereas the bulge height continues to gradually increase until the final hydroforming stage. This is related with the saturation behaviour that is generally exhibited by aluminium alloys and particularly, this AA6063 material. Also, the true stress vs. true strain curve is fitted using a Voce law and it emphasizes a highly saturation rate. Fig. 21 presents the experimental tube thickness at the pole dome plotted against the applied internal pressure. The results predicted by CB2001 are in good agreement with the experimental data.

Regarding to the variation of the pole thicknesses with respect to the bulge height, one can see that AA6063 presents a similar behaviour to the one observed for the S235 (see, Fig. 18). The thickness evolution plotted against the bulge height presents thereabouts a linear relationship, being fairly similar for all yield criteria, as shown in Fig. 22. Therefore, it can be deduced from curves displayed in Figs. 18 and 21 (for S235 and AA6063, respectively) that the linear evolution of the thickness distribution in the dome pole vs. the bulge height results from the strain path type experienced by this part of the tube during the free bulge test.

Fig. 23 depicts the numerical axial profiles computed using CB2001 yield criterion in the simulation of tube bulge test, along

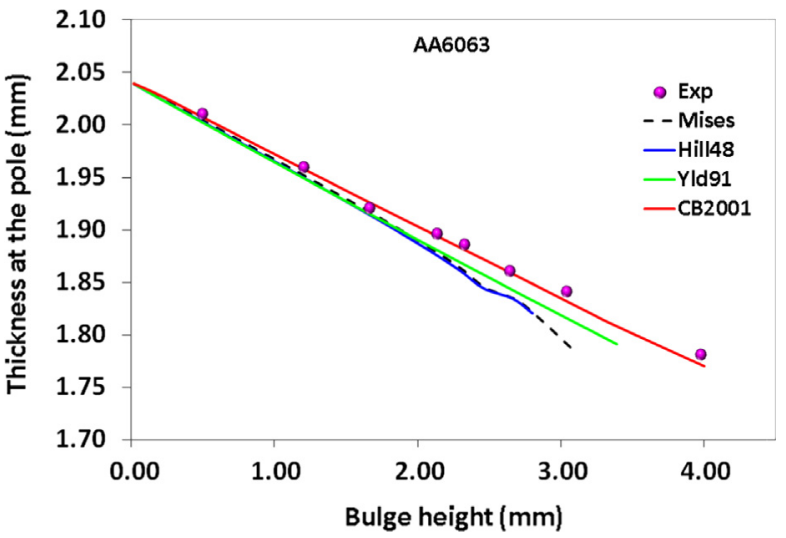

Fig. 22. Comparison between experimental and numerical thicknesses at the top of the pole vs. bulge height curves using the four yield criteria for AA6063 aluminium alloy.

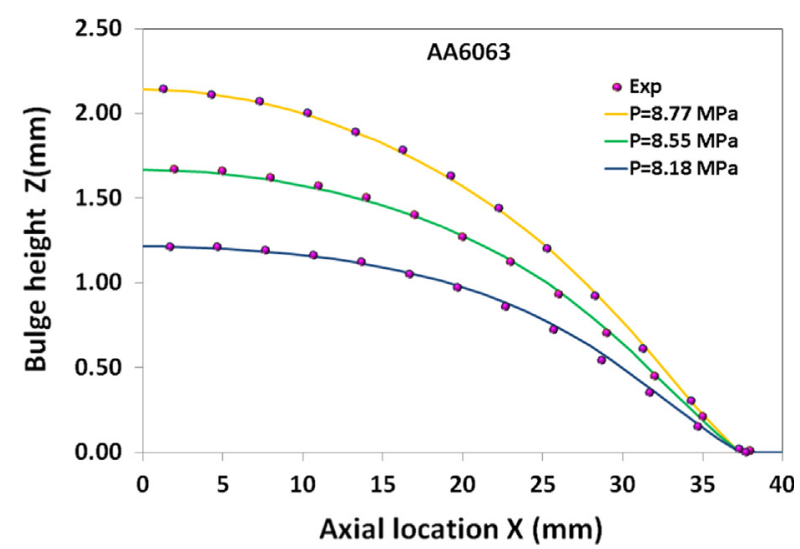

Fig. 23. Experimental and numerically predicted bulge profiles using the $\mathrm{CB} 2001$ yield criterion, for the AA6063 aluminium alloy.

with the experimental data obtained for the AA6063. These curves correspond to some specified bulge heights, which were obtained by applying the related pressure levels. These results emphasize good agreement with the experimental measurements. The profiles computed with Hill48 and Yld91 yield criteria are in drastic disagreement with the experimental profiles and therefore, they are not displayed here.

\section{Conclusions}

The mechanical behaviour of two anisotropic tubular materials was characterized by uniaxial tensile and free bulge tests using a home-designed hydroforming machine. Uniaxial tensile tests were performed to determine the tubular materials' yield stresses and anisotropy coefficients with respect to three directions, namely $0^{\circ}, 45^{\circ}$ and $90^{\circ}$ with respect to the referential direction; the free bulge test was also carried out to determine the biaxial stress state experienced by the bulged tubes at the pole dome. The identification of the anisotropy parameters of Hill48 and Yld91 yield criteria is performed using three yield stresses and three $r$-values obtained from uniaxial tensile tests, along with the biaxial stress state deduced from the free bulge test. However, this latter experimental data is insufficient for the identification of CB2001 anisotropy parameters. Thus, additional experimental input data is required and artificial input data is generated by Yld91, a less flexible but robust yield criterion, to substitute the missing experimental input data.

In order to assess the robustness of the proposed procedure for yield criteria calibration, the identified anisotropy parameters are 
used to simulate the tube bulge test. Numerical results such as the bulge height vs. internal pressure curves, thickness distribution and bulge profiles are obtained and compared to experimental data. Thereby, it is demonstrated that CB2001 yield criterion was successfully calibrated by combining reduced experimental results with artificial data. Moreover, CB2001 numerical predictions are indisputably the closest ones to the experimental results, mainly when compared to those obtained with Hill48 or Yld91 yield criteria. Nevertheless, Hill48 and Yld91 yield criteria can be useful to generate the so-called artificial input data which can be effectively employed as alternative to missing experimental data.

\section{Acknowledgements}

The authors gratefully acknowledge the financial support of the Portuguese Foundation for Science and Technology (FCT) via the projects PTDC/EMS-TEC/1805/2012 and PEst-C/EME/UI0285/2013 and by FEDER funds through the program COMPETE - Programa Operacional Factores de Competitividade, under the project CENTRO-07-0224-FEDER-002001 (MT4MOBI). The first author is also grateful for the Post-Doc grant.

\section{References}

[1] Ahmetoglu M, Altan T. Tube hydroforming: state of the art and future trends J Mater Process Technol 2000;98:25-33.

[2] Dohmann F, Hartl C. Hydroforming - a method to manufacture lightweight parts. J Mater Process Technol 1996;60:669-76.

[3] Bihamta R, D’Amours G, Bui Q-H, Guillot M, Raham A, Fafard M. Numerical and experimental studies on the new design concept of hydroforming dies for complex tubes. Mater Des 2013;47:766-78.

[4] Lang LH, Wang ZR, Kang DC. Hydroforming highlights: sheet hydro-forming and tube hydroforming. J Mater Process Technol 2004:151:165-77.

[5] Carleer B, Kevie G, Winter L, Veldhuizen B. Analysis of the effect of material properties on the hydroforming process of tubes. J Mater Process Technol 2000;104:158-66.

[6] Zhang SH. Developments in hydroforming. J Mater Process Technol 1998;91:236-44.

[7] Zribi T, Khalfallah A, Belhadjsalah H. A comparative study of the identification methods for tube hydroforming process. Key Eng Mater 2015;651-653:169_ 74.

[8] Saboori M, Champliaud H, Gholipour J, Gakwaya A, Savoie J, Wanjara P. Evaluation the flow stress of aerospace alloys for tube hydroforming process by free expansion testing. Int J Adv Manuf Technol 2014;72:1275-86.

[9] Koç M, Aul Yingyot, Altan T. On the characteristics of tubular materials for hydroforming - experimentation and analysis. Int J Mach Tool Manuf 2001; 14:761-72.

[10] Song WJ, Kim J, Kang BS. Experimental and analytical evaluation on flow stress of tubular material for tube hydroforming simulation. J Mater Process Technol 2007:191:368-71.

[11] Song WJ, Heo SC, Ku TW, Kim J, Kang BS. Evaluation of effect of flow stress characteristics of tubular material on forming limit in tube hydroforming process. Int J Mach Tool Manuf 2010;50:753-64.

[12] Green DE. Summary report of A/SP hydroforming work. Industrial Research of Development Institute; 2004.

[13] Songmene V, Bauwens C, Moses R. Free expansion hydroforming of AKDQ and HSLA steel tubes - experimental determination of circumferential stressstrain curves. A Report prepared by the Industrial Research \& Development Institute (IRDI) for the Hydroforming Materials and Lubricants task force of the Auto/Steel Partnership; 2000.

[14] Chen KK, Soldaat RJ, Moses RM. Free expansion bulge testing of tubes for automotive hydroform applications. SAE technical paper No. 2004-01-0832; 2004.

[15] Fuchizawa S, Narazaki M. Bulge test for determining stress-strain characteristics of thin tubes. In: Proceedings of the fourth ICTP advanced technology of plasticity 1993:488-93.

[16] Sokolowsky T, Gerke K, Ahmetgolu M, Altan T. Evaluation of tube formability and material characteristics: hydraulic bulge testing of tubes. J Mater Process Technol 2000;98:34-40.

[17] Hwang YM, Wang CW. Flow stress evaluation of zinc copper and carbon steel tubes by hydraulic bulge tests considering their anisotropy. J Mater Process Technol 2009;9:4423-8.
[18] Bortot P, Ceretti E, Giardini C. The determination of flow stress of tubular material for hydroforming applications. J Mater Process Technol 2008;203:381-8.

[19] Velasco R, Boudeau N, Michel G. Determination of tube hardening law using bulging tests. Int J Mater Form 2008;1:331-4.

[20] Lianfa Y, Cheng G. Determination of stress-strain relationship of tubular materials with hydraulic bulge test. Thin-Walled Struct 2008;46:147-54.

[21] Lui J, Liu X, Yang L, Liang H. Determination of flow stress of thin-walled tube based on digital speckle correlation method for hydroforming applications. Int J Adv Manuf Technol 2013;69:439-50.

[22] Fuchizawa S. Influence of plastic anisotropy on deformation of thin-walled tubes in bulge forming. In: Proceedings of the fourth ICTP advanced technology of plasticity 1987; vol. 2:727-32.

[23] Hwang YM, Lin YK, Altan T. Evaluation of tubular materials by a hydraulic bulge test. Int J Mach Tool Manuf 2007;47:343-51.

[24] Hwang YM, Lin YK. Analysis of tube forming in an open die considering anisotropic effects of the tubular material. Int J Mach Tool Manuf 2006;46:19218.

[25] Smith LM, Averill RC, Lucas JP. Double-sided high pressure tubular hydroforming. J Mater Process Technol 2003:142:599-608.

[26] Ben Ouirane AH, Boudeau N, Velasco R, Michel G. Error evaluation on experimental stress-strain curve obtained from tube bulging test. Thin-Walled Struct 2011;49:1217-24.

[27] Zribi T, Khalfallah A, Belhadjsalah $H$. Inverse method for flow stress parameters identification of tube hydroforming considering anisotropy. Int J Mech Manuf Syst 2011:4:441-53.

[28] Zribi T, Khalfallah A, Belhadjsalh H. Experimental characterization and inverse constitutive parameters identification of tubular materials for tube hydroforming process. Mater Des 2013;49:866-77.

[29] Strano M, Altan T. An inverse energy approach to determine the flow stress of tubular materials for hydroforming applications. J Mater Process Technol 2004; 104:92-6.

[30] Xu Y, Chan LC, Tsein YC, Gao L, Zheng PF. Prediction of work-hardening coefficient and exponential by adaptive inverse finite element method for tubular material. J Mater Process Technol 2008;201:413-8.

[31] Khalfallah A, Zribi T, Belhadjsalah H. Application of tube hydroforming using square cross-section die for inverse identification method validation. Key Eng Mater 2013;554-557:966-73.

[32] Hill R. The mathematical theory of plasticity. Oxford: Oxford University Press; 1950. p. $317-40$.

[33] Barlat F, Lege DJ, Brem JC. A six-component yield function for anisotropic materials. Int J Plast 1991;7:693-712.

[34] Cazacu O, Barlat F. Generalization of Drucker's yield criterion to orthotropy. Math Mech Solids 2001:613-30.

[35] Hershey AV. The plasticity of an isotropic aggregate of anisotropic face centred cubic crystals. J Appl Mech 1954;30:241.

[36] Hosford WF. A generalized isotropic yield function. J Appl Mech 1972;39:60719.

[37] Logan RW, Hosford WF. Upper-bound anisotropic yield locus calculations assuming (111)-pensil glide. Int J Mech Sci 1980;22:419-30.

[38] Drucker DC. Relation of experiments to mathematical theories of plasticity. J Appl Mech 1949;16:349-57.

[39] Alves JL, Oliveira MC, Menezes LF, Bouvier S. Influence of the yield criteria on the numerical results: the cross tool example. In: Proceedings of the 9th international conference on material forming, Esaform'06. Glasgow, UK; 2006.

[40] Barlat F, Brem JC, Yoon JW, Chung K, Dick RE, Choi SH, et al. Plane stress yield function for aluminium alloy sheets. Part 1. Theory. Int J Plast 2003:1297-319.

[41] Banabic D, Balan T, Comsa DS. A new yield criterion for orthotropic sheet metals under plane stress condition. In: TPR, Cluj-Napoca, Romania; 2000.

[42] Aydin MS, Gerlach J, Kessler L, Tekkaya AE. Yield locus evolution and constitutive parameter identification using plane strain tension and tensile tests. J Mater Process Technol 2011:1957-64.

[43] Aretz H, Hopperstad OS, Lademo O-G. Yield function calibration for orthotropic sheet metals based on uniaxial and plane stain tensile tests. J Mater Process Technol 2007;186:221-35.

[44] Jansson M, Nilsson L, Simonsson K. On constitutive modeling of aluminum alloys for tube hydroforming applications. Int J Plast 2005;21:1041-58.

[45] Khalfallah A, Alves JL, Oliveira MC, Menezes LF. Influence of the characteristics of the experimental data set used to identify anisotropy parameters. Simul Modell Pract Theory 2015;53:15-44.

[46] Alves JL, Oliveira MC, Menezes LF. Estratégias de identificaçao de parâmetros constituvos. In: Proceedings of the congresso de métodos computacionais em engenharia. Lisbon; June 2004.

[47] Menezes LF, Teodosiu C. Three-dimentional numerical simulation of deepdrawing process using solid finite elements. J Mater Process Technol 2000;97:100-6.

[48] Koç M, Allen T, Jiratheranat S, Altan T. The use of FEA and design of experiments to establish design guidelines for simple hydroformed parts. Int J Mach Tool Manuf 2000;40:2249-66. 